\title{
PROFINITE COMPLETIONS OF BURNSIDE-TYPE QUOTIENTS OF SURFACE GROUPS
}

\author{
LOUIS FUNAR AND PIERRE LOCHAK
}

\begin{abstract}
Using quantum representations of mapping class groups we prove that profinite completions of Burnside-type surface group quotients are not virtually prosolvable, in general. Further, we construct infinitely many finite simple characteristic quotients of surface groups.
\end{abstract}

2000 MSC Classification: 57 M 07, 20 F 36, 20 G 20, 22 E 40.

Keywords: Mapping class group, Burnside group, quantum representation, discrete subgroup of semi-simple Lie groups, congruence quotient, characteristic subgroup.

\section{InTRODUCTION AND STATEMENTS}

Let $\pi_{g}$ denote the fundamental group $\pi_{1}\left(S_{g}, p\right)$ of a closed orientable surface $S_{g}$ of genus $g$, based at a point $p \in S_{g}$. Recall that $\pi_{g}$ is a one-relator group with the presentation:

$$
\pi_{g}=\left\langle a_{1}, a_{2}, \ldots, a_{g}, b_{1}, b_{2}, \ldots, b_{g} \mid\left[a_{1}, b_{1}\right] \cdots\left[a_{2}, b_{2}\right] \cdots\left[a_{g}, b_{g}\right]=1\right\rangle
$$

Here the classes $a_{i}, b_{i}$ are represented by non-separating simple closed loops on $S_{g}$ based at $p$.

We denote by $\Gamma_{g}$ the mapping class group of $S_{g}$. Further $\Gamma_{g}^{1}$ denotes the mapping class group of the pair $\left(S_{g}, p\right)$, namely the group of isotopy classes of orientation preserving homeomorphisms of $S_{g}$ fixing $p$. It is well-known that $\Gamma_{g}^{1}$ is isomorphic to the mapping class group of the punctured surface $S_{g}-\{p\}$. By forgetting the marked point $p$ one obtains a surjective homomorphism $\Gamma_{g}^{1} \rightarrow \Gamma_{g}$ which is part of the Birman exact sequence:

$$
1 \rightarrow \pi_{g} \rightarrow \Gamma_{g}^{1} \rightarrow \Gamma_{g} \rightarrow 1
$$

The Dehn-Nielsen-Baer theorem states that the map associating to $\varphi \in \Gamma_{g}^{1}$ the automorphism $\varphi_{*}: \pi_{1}\left(S_{g}, p\right) \rightarrow \pi_{1}\left(S_{g}, p\right)$ provides an isomorphism between $\Gamma_{g}^{1}$ and $\operatorname{Aut}^{+}\left(\pi_{g}\right)$ and induces an isomorphism $\Gamma_{g} \rightarrow \mathrm{Out}^{+}\left(\pi_{g}\right)$. Furthermore, the diagram below is commutative:

$$
\begin{aligned}
& 1 \rightarrow \pi_{g} \rightarrow \Gamma_{g}^{1} \quad \rightarrow \quad \Gamma_{g} \quad \rightarrow \quad 1
\end{aligned}
$$

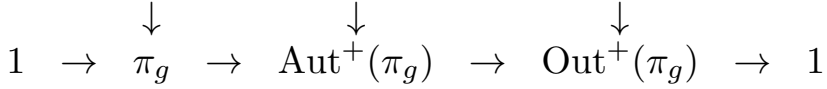

where the top horizontal line is the Birman exact sequence.

If $M \subset \pi_{g}$ we denote by $M[n]$ the normal subgroup of $\pi_{g}$ generated by $\varphi_{*}\left(x^{n}\right)$, for all $x \in M$ and $\varphi_{*} \in \operatorname{Aut}^{+}\left(\pi_{g}\right)$. Note that $M[n]$ is the characteristic subgroup generated by the subset $M^{n}$ of $n$-th powers of elements in $M$.

The Burnside-type group $B\left(\pi_{g}, n, M\right)$ is the quotient $\pi_{g} / M[n]$. Several choices for $M$ are particularly interesting. An element $x \in \pi_{g}$ is called primitive if it can be represented by a non-separating simple closed curve on $S_{g}$. This is equivalent to saying (see [45]) that $x \in \pi_{g}$ can be mapped into one generator, say $a_{1}$, by some automorphism $\varphi_{*} \in \operatorname{Aut}^{+}\left(\pi_{g}\right)$, where $a_{1}, \ldots, a_{g}, b_{1}, \ldots, b_{g}$ are the generators from the standard presentation above.

The set of primitive classes of $\pi_{g}$ is then contained in the set $\mathcal{S}\left(S_{g}\right)$ of homotopy classes of simple closed curves on $S_{g}$. More generally, we set $\mathcal{S}_{n}\left(S_{g}\right)$ for the set of homotopy classes of closed curves on $S_{g}$ with at most $n$ self-intersections.

We denote by $\widehat{G}$ the profinite completion of a group $G$. We are concerned in this paper with how large the profinite completion of $B\left(\pi_{g}, n, M\right)$ could be. Our first result is: 
Theorem 1.1. Let $g \geq 2$ and $p \equiv 3(\bmod 4)$ a large enough prime. Then for every $m$ there exists some $d$ such that the group $B\left(\widehat{\pi_{g}, d p}, M\right)$ is not virtually prosolvable, if $M \subset \mathcal{S}_{m}\left(S_{g}\right)$. When $m=1$ then $d=1$.

Remark 1.1. The result above also holds for large enough primes $p \equiv 1(\bmod 4)$, according to Remark 2.1. An explicit $p_{0}$ such that the claim holds for all $p \geq p_{0}$ can be obtained from effective bounds in Lemma 3.8. Moreover, the claim holds for all primes $p<10^{4}$, by a computer check of Lemma 3.8 ,

The proof shows that under these assumptions $B\left(\widehat{\pi_{g}, d p}, M\right)$ is neither solvable-by-finite nor finite-by-solvable. Our notational convention is that a finite-by-solvable group is an extension of a finite group by some solvable group, also called a virtually solvable group in the litterature.

Zelmanov [45] considered the group $\widehat{\pi_{g}} /\langle M[n]\rangle$, where $\left\langle M^{n}\right\rangle$ is the closure in $\widehat{\pi_{g}}$ of the normal subgroup of $\widehat{\pi_{g}}$ generated by $M^{n}$. Problem 2 from [45] asked whether this group is solvable-byfinite, when $M$ denotes the set of primitive elements of $\pi_{g}$. The result above shows that this is not the case, in general:

Corollary 1.2. Let $g \geq 2$ and $p \equiv 3(\bmod 4)$, a large enough prime. The group $\widehat{\pi_{g}} /\langle M[n]\rangle$, where $M=\mathcal{S}\left(S_{g}\right)$ contains the set of primitive elements of $\pi_{g}$, is not virtually solvable.

Proof. The surjective map $\pi_{g} \rightarrow B\left(\pi_{g}, p, M\right)$ induces a surjective continuous homomorphism between the corresponding profinite completions $\widehat{\pi_{g}} \rightarrow B\left(\widehat{\pi_{g}, p,} M\right)$. The kernel of the last map contains $M[n]$ and hence the closure $\left\langle M^{n}\right\rangle$ of the normal subgroup of $\widehat{\pi_{g}}$ generated by $M^{n}$. Therefore we have a surjective continuous map $\widehat{\pi_{g}} /\langle M[n]\rangle \rightarrow B\left(\widehat{\pi_{g}, p, M}\right)$.

Our method also provides a large supplies of finite quotients for all intermediary subgroups:

Corollary 1.3. Let $g \geq 2$ and $\Gamma$ be a group such that $\pi_{g} \subset \Gamma \subset \Gamma_{g}^{1}$. Then $\Gamma$ admits surjective homomorphisms onto infinitely many finite simple groups, for instance $\operatorname{PSL}\left(N, \mathbf{F}_{q}\right)$, where $N$ and $q$ are arbitrarily large.

In particular, this holds if $\Gamma$ is the fundamental group of a closed 3-manifold fibering over the circle with fiber a closed orientable surface of genus $g \geq 2$.

Recall that a subgroup $H \subset G$ is characteristic if it is invariant by the action of the group $\operatorname{Aut}(G)$ of automorphisms of $G$. Further, the quotient $Q$ of $G$ is a characteristic quotient of $G$ if there is a surjective homomorphism $p: G \rightarrow Q$ whose kernel ker $p$ is a characteristic subgroup of $G$. A consequence of an intermediary result obtained in the proof of Theorem 1.1 is the following:

Theorem 1.4. For $g \geq 2$ there exist infinitely many finite simple characteristic quotients of $\pi_{g}$.

This answers a question of Lubotzky from ([22], sections 10 and 6.4).

The proof of the main results goes as follows. We consider the so-called quantum representations of the mapping class groups $\Gamma_{g}$ and $\Gamma_{g}^{1}$ depending on some root of unity of order $2 p$. It was proved in [10] that these representations have infinite image, for $p \geq 5$. The proof was simplified in [27] where explicit elements of infinite order were found. Further, in [21] the authors showed that the images of $\Gamma_{g}$ are topologically dense in the corresponding special unitary groups, when $p \geq 5$ is prime. On the other hand the matrices in the images have coefficients in a cyclotomic ring (see [15]). Eventually the restriction of scalars provides Zariski dense discrete representations in semisimple linear algebraic groups defined over $\mathbb{Q}$ whose images are contained in arithmetic groups of higher rank (see [15]). The aim of [11] and [28] was to construct quotients of $\Gamma_{g}$ which are simple finite groups of Lie type of arbitrary large rank.

Our strategy here is to consider the restriction of the quantum representations from $\Gamma_{g}^{1}$ to the subgroup $\pi_{g}$. These representations were recently studied by Koberda and Santharoubane in [18], where it is proved that they still have infinite images, while they factor through the Burnside-type group $B\left(\pi_{g}, p, \mathcal{S}\left(S_{g}\right)\right)$. Our aim is to show that the restriction of scalars provides Zariski dense discrete representations of $B\left(\pi_{g}, p, \mathcal{S}\left(S_{g}\right)\right)$ in some semi-simple linear algebraic group defined over $\mathbb{Q}$ of higher $\mathbb{R}$-rank. The Nori-Weisfeiler approximation theorem (see [30, 44]) then provides many finite quotients of congruence type. This implies that our profinite Burnside-type groups surjects onto an infinite product of simple non-abelian groups, proving our first theorem. We note that the 
image of the surface group coincides with that of the mapping class group. Thus every kernel of a homomorphism of $\pi_{g}$ onto a finite simple quotient obtained this way is invariant by the mapping class group action. We obtain therefore finite simple characteristic quotients of $\pi_{g}$, proving the second theorem. Eventually, we notice that the quotients obtained by this method are principal congruence quotients.

Acknowledgements. The authors are indebted to C. Blanchet, S. Checcoli, P. Eyssidieux, T. Koberda, J. Marché, B. Remy and T. Venkataramana for useful discussions and to the referee for several corrections, suggestions improving the presentation and for pointing out that our methods also apply to Lubotzky's question above.

\section{Preliminaries on QUANTUM MAPPING Class Group RePresentations}

2.1. The setting of the skein TQFT. A TQFT is a functor from the category of surfaces into the category of finite dimensional vector spaces. Specifically, the objects of the first category are closed oriented surfaces endowed with colored banded points and morphisms between two objects are cobordisms decorated by uni-trivalent ribbon graphs compatible with the banded points. A banded point on a surface is a point with a tangent vector at that point, or equivalently a germ of an oriented interval embedded in the surface. There is a corresponding surface with colored boundary obtained by deleting a small neighborhood of the banded points and letting the boundary circles inherit the colors of the respective points.

We will use the TQFT functor $\mathcal{V}_{p}$, for $p \geq 3$ and a primitive root of unity $A$ of order $2 p$, as defined in [2]. The vector space associated by the functor $\mathcal{V}_{p}$ to a surface is called the space of conformal blocks. Let $S_{g}$ denote the genus $g$ closed orientable surface, $H_{g}$ be a genus $g$ handlebody with $\partial H_{g}=\Sigma_{g}$. Assume given a finite set $\mathcal{Y}$ of banded points on $S_{g}$. Let $G$ be a uni-trivalent ribbon graph embedded in $H_{g}$ in such a way that $H_{g}$ retracts onto $G$, its univalent vertices are the banded points $\mathcal{Y}$ and it has no other intersections with $S_{g}$.

We fix a natural odd number $p \geq 3$, called the level of the TQFT. We define the set of colors in level $p$ to be $\mathcal{C}_{p}=\{0,2,4, \ldots, p-3\}$.

An edge coloring of $G$ is called $p$-admissible if the triangle inequality is satisfied at any trivalent vertex of $G$ and the sum of the three colors around a vertex is bounded by $2(p-2)$.

Fix a coloring of the banded points $\mathcal{Y}$. Then there exists a basis of the space of conformal blocks associated to the surface $\left(\Sigma_{g}, \mathcal{Y}\right)$ with the colored banded points (or the corresponding surface with colored boundary) which is indexed by the set of all $p$-admissible colorings of $G$ extending the boundary coloring. We denote by $W_{g,\left(i_{1}, i_{2}, \ldots, i_{r}\right)}$ the vector space associated to the closed surface $\Sigma_{g}$ with $r$ banded points colored by $i_{1}, i_{2}, \ldots, i_{r} \in \mathcal{C}_{p}$. Note that banded points colored by 0 do not contribute.

Observe that an admissible $p$-coloring of $G$ provides an element of the skein module $S_{A}\left(H_{g}\right)$ of the handlebody with banded boundary points colored $\left(i_{1}, i_{2}, \ldots, i_{r}\right)$, evaluated at the primitive $2 p$-th root of unity $A$. This skein element is obtained by cabling the edges of $G$ by the Jones-Wenzl idempotents prescribed by the coloring and having banded points colors fixed. We suppose that $H_{g}$ is embedded in a standard way into the 3 -sphere $S^{3}$, so that the closure of its complement is also a genus $g$ handlebody $\bar{H}_{g}$. There is then a sesquilinear form:

$$
\langle,\rangle: S_{A}\left(H_{g}\right) \times S_{A}\left(\bar{H}_{g}\right) \rightarrow \mathbb{C}
$$

defined by

$$
\langle x, y\rangle=\langle x \sqcup y\rangle \text {. }
$$

Here $x \sqcup y$ is the element of $S_{A}\left(S^{3}\right)$ obtained by the disjoint union of $x$ and $y$ in $H_{g} \cup \bar{H}_{g}=S^{3}$, and \langle\rangle$: S_{A}\left(S^{3}\right) \rightarrow \mathbb{C}$ is the Kauffman bracket invariant.

Eventually the space of conformal blocks $W_{g,\left(i_{1}, i_{2}, \ldots, i_{r}\right)}$ is the quotient $S_{A}\left(H_{g}\right) / \operatorname{ker}\langle$,$\rangle by the$ left kernel of the sesquilinear form above. It follows that $W_{g,\left(i_{1}, i_{2}, \ldots, i_{r}\right)}$ is endowed with an induced Hermitian form $H_{A}$.

The projections of skein elements associated to the $p$-admissible colorings of a trivalent graph $G$ as above form an orthogonal basis of $W_{g,\left(i_{1}, i_{2}, \ldots, i_{r}\right)}$ with respect to $H_{A}$. It is known ([2]) that $H_{A}$ only depends on the $p$-th root of unity $\zeta_{p}=A^{2}$ and that in this orthogonal basis the diagonal entries belong to the totally real maximal subfield $\mathbb{Q}\left(\zeta_{p}+\overline{\zeta_{p}}\right)$ (after rescaling). 
Let $G^{\prime} \subset G$ be a uni-trivalent subgraph whose degree one vertices are colored, corresponding to a sub-surface $\Sigma^{\prime}$ of $\Sigma_{g}$ with colored boundary. The projections in $W_{g,\left(i_{1}, i_{2}, \ldots, i_{r}\right)}$ of skein elements associated to the $p$-admissible colorings of $G^{\prime}$ form an orthogonal basis of the space of conformal blocks associated to the surface $\Sigma^{\prime}$ with colored boundary components.

There is a geometric action of the mapping class groups of the handlebodies $H_{g}$ and $\bar{H}_{g}$ respectively on their skein modules and hence on the space of conformal blocks. Moreover, these actions extend to a projective action $\rho_{g, p,\left(i_{1}, \ldots, i_{r}\right), A}$ of $\Gamma_{g}^{r}$ on $W_{g,\left(i_{1}, i_{2}, \ldots, i_{r}\right)}$ respecting the Hermitian form $H_{\zeta_{p}}=H_{A}$. When referring to $\rho_{g, p,\left(i_{1}, \ldots, i_{r}\right), A}$ the subscript specifying the genus $g$ will most often be dropped when its value will be clear from the context. Notice that the mapping class group of an essential (i.e. without annuli or disks complements) sub-surface $\Sigma^{\prime} \subset \Sigma_{g}$ is a subgroup of $\Gamma_{g}$ which preserves the subspace of conformal blocs associated to $\Sigma^{\prime}$ with colored boundary. It is worthy to note that $\rho_{p,\left(i_{1}, \ldots, i_{r}\right), A}$ only depends on $\zeta_{p}=A^{2}$, so we can unambigously shift the notation for this representation to $\rho_{p,\left(i_{1}, \ldots, i_{r}\right), \zeta_{p}}$.

There is a central extension $\widetilde{\Gamma_{g}}$ of $\Gamma_{g}$ by $\mathbb{Z}$ and a linear representation $\widetilde{\rho}_{p, \zeta_{p}}$ on $W_{g}$ which resolves the projective ambiguity of $\rho_{p, \zeta_{p}}$. The largest such central extension has class 12 times the Euler class (see [14, 29]), but the central extension considered in this paper is an index 12 subgroup of it, called $\widetilde{\Gamma}_{1}$ in [29]. When $g \geq 3$ it is a perfect group which therefore coincides with the universal central extension.

We denote by $S_{g, n}^{r}$ the compact orientable surface of genus $g$ with $n$ boundary components and $r$ marked points. Then $\Gamma_{g, n}^{r}$ denotes the pure mapping class group of $S_{g, n}^{r}$ which fixes pointwise boundary components and marked points.

We consider a subsurface $\Sigma_{g, r} \subset \Sigma_{g+r}$ whose complement consists of $r$ copies of $\Sigma_{1,1}$. Let $\widetilde{\Gamma_{g}^{r}}$ be the pull-back of the central extension $\widetilde{\Gamma_{g}}$ to the subgroup $\Gamma_{g, r} \subset \Gamma_{g+r}$. Then $\widetilde{\Gamma_{g, r}}$ is also a central extension, which we denote $\widetilde{\Gamma_{g}^{r}}$ of $\Gamma_{g}^{r}$ by $\mathbb{Z}^{r+1}$. From [14, 29] we derive that $\widetilde{\Gamma_{g}^{r}}$ is perfect, when $g \geq 3$ and of order 10 , when $g=2$.

Definition 2.1. Let $p \geq 5$ be odd and $\zeta_{p}$ a primitive $p$-th root of unity. We denote by $\widetilde{\rho}_{p, \zeta_{p},\left(i_{1}, i_{2}, \ldots, i_{r}\right)}$ the linear representation of the central extension $\widetilde{\Gamma_{g}^{r}}$ which acts on the vector space $W_{g, p,\left(i_{1}, i_{2}, \ldots, i_{r}\right)}$ associated by the TQFT to the surface with the corresponding colored banded points (see [14, 29]).

The functor $\mathcal{V}_{p}$ associates to a handlebody $H_{g}$ the projection of the skein element corresponding to the trivial coloring of the trivalent graph $G$ by 0 . The invariant associated to a closed 3-manifold is given by pairing the two vectors associated to handlebodies in a Heegaard decomposition of some genus $g$ and taking into account the twisting by the gluing mapping class action on $W_{g}$.

One should notice that the skein TQFT $\mathcal{V}_{p}$ is unitary, in the sense that $H_{\zeta_{p}}$ is a positive definite Hermitian form when $\zeta_{p}=(-1)^{p} \exp \left(\frac{2 \pi i}{p}\right)$, corresponding to $A_{p}=(-1)^{\frac{p-1}{2}} \exp \left(\frac{(p+1) \pi i}{2 p}\right)$. For the sake of notational simplicity, from now we will drop the subscript $p$ in $\zeta_{p}$, when the order of the root of unity will be clear from the context and the precise choice of the root of given order won't matter. Note that for a general primitive $p$-th root of unity, the isometries of $H_{\zeta}$ form a pseudo-unitary group.

Now, the image $\rho_{p, \zeta}\left(T_{\gamma}\right)$ of a right hand Dehn twist $T_{\gamma}$ in a convenient basis given by a trivalent graph is easy to describe. Assume that the simple curve $\gamma$ is the boundary of a small disk intersecting once transversely an edge $e$ of the graph $G$. Consider $v \in W_{g}$ be a vector of the basis given by colorings of the graph $G$ and assume that edge $e$ is labeled by the color $c(e) \in \mathcal{C}_{p}$. Then the action of the (canonical) lift $\widetilde{T_{\gamma}}$ of the Dehn twist $T_{\gamma}$ in $\widetilde{\Gamma_{g}}$ is given by (see [2, 5.8) :

$$
\widetilde{\rho}_{p, \zeta}\left(\widetilde{T_{\gamma}}\right) v=A^{c(e)(c(e)+2)} v
$$

2.2. Unitary groups of spaces of conformal blocks. For a prime $p \geq 5$ we denote by $\mathcal{O}_{p}$ the ring of cyclotomic integers $\mathcal{O}_{p}=\mathbb{Z}\left[\zeta_{p}\right]$, if $p \equiv 3(\bmod 4)$ and $\mathcal{O}_{p}=\mathbb{Z}\left[\zeta_{4 p}\right]$, if $p \equiv 1(\bmod 4)$ respectively, where $\zeta_{r}$ denotes a primitive $r$-th root of unity (the subscript $r$ will sometimes be omitted). The main result of [15] states that there exists a free $\mathcal{O}_{p}$-lattice $\Lambda_{g, p}$ in the $\mathbb{C}$-vector space of conformal blocks associated by the TQFT $\mathcal{V}_{p}$ to the genus $g$ closed orientable surface and a non-degenerate Hermitian $\mathcal{O}_{p}$-valued form on $\Lambda_{g, p}$ both invariant under the action of $\widetilde{\Gamma_{g}}$ via the representation $\widetilde{\rho}_{p, \zeta}$. Therefore the image of the mapping class group consists of unitary matrices (with respect to 
the Hermitian form) with entries in $\mathcal{O}_{p}$. Let $\mathbb{U}_{g, p, \zeta}\left(\mathcal{O}_{p}\right)$ and $P \mathbb{U}_{g, p, \zeta}\left(\mathcal{O}_{p}\right)$ be the group of all such matrices and respectively its quotient by scalars. Then $\mathbb{U}_{g, p, \zeta}\left(\mathcal{O}_{p}\right)$ is the group of $\mathcal{O}_{p}$-points of the unitary group $\mathbb{U}_{g, p, \zeta}$ associated to the Hermitian form $H_{\zeta}$, which is a linear algebraic group defined over $\mathbb{Q}(\zeta+\bar{\zeta})$.

When $p$ is prime $p \geq 5$ and $g \geq 2,(g, p) \neq 5$, then $\widetilde{\rho}_{p, \zeta_{p}}$ takes values in the special unitary group $S \mathbb{U}_{g, p, \zeta_{p}}$ (see [5, 11, 12]). It is known that $S \mathbb{U}_{g, p, \zeta}\left(\mathcal{O}_{p}\right)$ is an irreducible lattice in a semi-simple algebraic group $\mathbb{G}_{g, p}$ obtained by the so-called restriction of scalars construction from the totally real cyclotomic field $\mathbb{Q}\left(\zeta_{p}+\overline{\zeta_{p}}\right)$ to $\mathbb{Q}$. Specifically, the group $\mathbb{G}_{g, p}$ is a product $\prod_{\sigma \in S(p)} S \mathbb{U}_{g, p, \sigma(\zeta)}$. Here $S(p)$ stands for a set of representatives of the classes of complex embeddings $\sigma$ of $\mathcal{O}_{p}$ modulo complex conjugation, or equivalently the set of places of the totally real cyclotomic field $\mathbb{Q}\left(\zeta_{p}+\overline{\zeta_{p}}\right)$. The factor $S \mathbb{U}_{g, p, \sigma(\zeta)}$ is the special unitary group associated to the Hermitian form conjugated by $\sigma$, thus corresponding to some Galois conjugate root of unity.

Denote by $\widetilde{\rho}_{p}$ and $\rho_{p}$ the representations $\prod_{\sigma \in S(p)} \widetilde{\rho}_{p, \sigma\left(A_{p}^{2}\right)}$ and $\prod_{\sigma \in S(p)} \rho_{p, \sigma\left(A_{p}^{2}\right)}$, respectively. Notice that the real Lie group $\mathbb{G}_{g, p}$ is a semi-simple algebraic group defined over $\mathbb{Q}$.

In [11] it is proved that $\widetilde{\rho}_{p}\left(\widetilde{\Gamma_{g}}\right)$ is a discrete Zariski dense subgroup of $\mathbb{G}_{g, p}(\mathbb{R})$ whose projections onto the simple factors of $\mathbb{G}_{g, p}(\mathbb{R})$ are topologically dense, for $g \geq 3$ and $p \geq 7$ prime, $p \equiv 3(\bmod 4)$.

Remark 2.1. When $p \equiv 1(\bmod 4)$ the image of the central extension of $\Gamma_{g}$ from [29] by $\widetilde{\rho}_{p}$ is contained in $\mathbb{G}_{g, p}(\mathbb{Z}[i])$ and thus it is a discrete Zariski dense subgroup of $\mathbb{G}_{g, p}(\mathbb{C})$. However, if we restrict to the universal central extension $\widetilde{\Gamma_{g}}$ coefficients are reduced from $\mathbb{Z}\left[\zeta_{4 p}\right]$ to $\mathbb{Z}\left[\zeta_{p}\right]$ (see $[15$, section 13). Note that the corresponding invariant form $H_{\zeta_{p}}$ should be suitably rescaled and after rescaling it will be skew-Hermitian when $g$ is odd and Hermitian for even $g$.

As mentioned in ([28], Rem.3.5) for the proof of our main result we don't need the integral TQFT of [15] as the Burnside-type groups are finitely generated and hence only finitely primes could appear in the denominators of matrices in their image.

\section{QuANTUM SURFACE GROUP REPRESENTATIONS}

3.1. Zariski density of quantum representations. Our aim is to find the Zariski closures of $\rho_{p,(i)}\left(\pi_{g}\right)$. We follow closely the strategy from [11, where we proved that $\rho_{p,(i)}\left(\Gamma_{g}\right)$ is Zariski dense in $P \mathbb{G}_{p}(\mathbb{R})$, based on the topological density result in the corresponding special unitary group earlier obtained in [21].

The mapping class group $\Gamma_{g, 1}$ is a subgroup of $\Gamma_{g+1}$, by identifying $S_{g+1}$ with the result of gluing of $S_{g, 1}$ and $S_{1,1}$. It is well-known that

$$
W_{g+1, p}=\bigoplus_{i} W_{g, p,(i)} \otimes W_{1, p,(i)}
$$

The decomposition corresponds to the eigenspaces for the Dehn twist $\widetilde{T}_{c}$ along the curve $c=\partial S_{g, 1}$. Let $\mathbb{U}_{g, p, \zeta,(i)}=U\left(W_{g, p,(i)}, H_{\zeta}\right)$ be the unitary subgroup keeping invariant the subspace $W_{g, p,(i)}$, when endowed with the (restriction of the) Hermitian form $H_{\zeta}$. The group $\mathbb{U}_{g, p, \zeta,(i)}$ is a closed linear algebraic subgroup of $\mathbb{U}_{g+1, p, \zeta}$ and is also defined over the maximal totally real algebraic field $\mathbb{Q}(\zeta+\bar{\zeta})$ of $\mathbb{Q}(\zeta)$.

Since $\widetilde{\Gamma_{g}^{1}}$ is perfect when $g \geq 3$ and of order 10 for $g=2$, it follows that $\widetilde{\rho}_{p,(i)}\left(\widetilde{\Gamma_{g}^{1}}\right)$ is contained within the special unitary group $S \mathbb{U}_{g, p, \zeta,(i)}$, if $(g, p) \neq(2,5)$, as in [5, 11, 12].

We denote by $\mathbb{G}_{g, p,(i)}$ the group obtained by scalar restriction from $\mathbb{Q}(\zeta+\bar{\zeta})$ to $\mathbb{Q}$ of the linear algebraic group $S \mathbb{U}_{g, p, \zeta,(i)}$, namely the product $\mathbb{G}_{g, p,(i)}=\prod_{\sigma \in S(p)} S \mathbb{U}_{g, p, \sigma(\zeta),(i)}$. It follows that the product representation $\widetilde{\rho}_{p,(i)}=\prod_{\sigma \in S(p)} \widetilde{\rho}_{p, \sigma\left(A_{p}^{2}\right),(i)}$ of $\widetilde{\Gamma_{g}^{1}}$ takes values in $\mathbb{G}_{g, p,(i)}$. Since the boundary Dehn twist acts as a scalar this representation of $\widetilde{\Gamma_{g}^{1}}$ descends to a projective representation $\rho_{g, p,(i)}$ : $\Gamma_{g}^{1} \rightarrow P \mathbb{G}_{g, p,(i)}$.

Set $\widetilde{\pi_{g}}=\operatorname{ker}\left(\widetilde{\Gamma_{g}^{1}} \rightarrow \Gamma_{g}\right)$. It follows that $\widetilde{\pi_{g}}$ is an extension by $\mathbb{Z}^{2}$ of $\pi_{g}$.

Our main result in this section is: 
Theorem 3.1. Let $g \geq 2$ and $p \equiv 3(\bmod 4), p$ a large enough prime. Then the Zariski closure of $\widetilde{\rho}_{p,(p-3)}\left(\widetilde{\pi_{g}}\right)$ is $\mathbb{G}_{g, p,(p-3)}(\mathbb{R})$. Moreover, if $g \geq 3$ every non-compact factor of $\mathbb{G}_{g, p,(p-3)}(\mathbb{R})$ has real rank at least 2 .

The rest of this section is devoted to the proof of the theorem above.

The key ingredient is the following proposition, whose rather technical proof is postponed to section 3.3 below:

Proposition 3.1. Let $g \geq 2$ and $p \equiv 3(\bmod 4), p$ a large enough prime. The representation $\widetilde{\rho}_{p, \zeta,(p-3)}$ of $\widetilde{\Gamma_{g, 1}}$ into $W_{g, p,(p-3)}$ has dense image in the special unitary group $S \mathbb{U}_{g, p, \zeta,(p-3)}$.

Proposition 3.2. Let $g \geq 2$ and $p \geq 5$ be odd. Suppose that $\widetilde{\rho}_{p, \zeta,(i)}\left(\widetilde{\Gamma_{g, 1}}\right)$ is Zariski dense in $S \mathbb{U}_{g, p, \zeta,(i)}$. Then $\widetilde{\rho}_{p, \zeta,(i)}\left(\widetilde{\pi_{g}}\right)$ is Zariski dense in the special unitary group $S \mathbb{U}_{g, p, \zeta,(i)}$.

Proof of Proposition 3.2. As $\pi_{g}$ is a normal subgroup of $\Gamma_{g}^{1}$, we derive that the topological closure of its image by $\rho_{p, \zeta,(i)}$ is a closed normal Lie subgroup of the projective unitary group $P \mathbb{U}_{g, p, \zeta,(i)}$. Therefore the image of $\widetilde{\pi_{g}}$ is a closed normal subgroup of $S \mathbb{U}_{g, p, \zeta,(i)}$. Since the Lie algebra of $S \mathbb{U}_{g, p, \zeta,(i)}$ is simple it follows that the Lie group has dimension zero and hence it is a discrete subgroup. However a normal discrete subgroup of $S \mathbb{U}_{g, p, \zeta,(i)}$ must be contained in its center, which is cyclic of order $\operatorname{dim} W_{g, p,(i)}$.

Now, the result of [18] for $i=2$ shows that the image of $\pi_{g}$ by $\rho_{p, \zeta,(i)}$ is infinite non-abelian. We claim that this holds true for all $i \neq 0$ and we will give a detailed proof for $i=p-3$.

The $k+1$-holed sphere $S_{0, k+1}$, whose boundary circles are colored by $\mathbf{c}=(a, a, \ldots, a, a k-2)$ has associated a space of conformal blocks $W_{0, \mathbf{c}}$ of dimension $k$ which has a natural action of the braid group $B_{k}$ on $k$ strings. Note that $\Sigma_{0, k+1}$ can be embedded into $\Sigma_{g, 1}$ such that the homomorphism $B_{k} \rightarrow \Gamma_{g, 1}$ is injective, if $k \leq g$. It is well-known that this braid group action coincides with the Burau representation at a suitable root of unity (see [13]) twisted by a character. Specifically, the Burau representation is the one for which the standard braid generators have eigenvalues -1 and $A_{p}^{2 a^{2}}$. Moreover, in [13] one proved that the image of the Burau representation of $B_{3}$ is infinite non-abelian if $A_{p}^{2 a^{2}}$ is not a primitive root of unity of order $2,3,4$ or 5 , while the image of $B_{4}$ is infinite non-abelian (see e.g. [10]) if $A_{p}^{2 a^{2}}$ is not a primitive root of unity of order 2 or 3 .

It suffices to consider $i \geq 4$. If we can write $i=a k-2,3 \leq k \leq g, a \in \mathcal{C}_{p}$, the image of $B_{k}$ is infinite. Further $\pi_{1}\left(S_{0,3}\right) \subset \pi_{1}\left(S_{0, k+1}\right)$, if $k \geq 2$ and the restriction of the Burau representation to the pure braid group $P B_{3}$ is infinite non-abelian. But $P B_{3}=\mathbb{F}_{2} \times \mathbb{Z}$, where the factor $\mathbb{Z}$ is central and its image by the Burau representation is of finite order, while the free factor $\mathbb{F}_{2}$ can be identified with $\pi_{1}\left(S_{0,3}\right)$. We derive that the image of $\pi_{1}\left(S_{g, 1}\right)$ by the subrepresentation of $\rho_{p,(i)}$ corresponding to the Burau representation contains the image of $\mathbb{F}_{2}$, namely a triangle group according to [13.

When $g=2$ and $i=p-3$ we consider the image of $\pi_{1}\left(\Sigma_{1,2}\right)$ by the quantum representation of $\Gamma_{2}^{1}$, where $\Sigma_{1,2} \subset \Sigma_{2,1}$ is the complementary of a one holed torus with boundary label 2 . Then $\Gamma_{1,2}$ acts on $W_{1, p,(2, p-3)}$ which has dimension 3 and an explicit calculation shows that the image of $\pi_{1}\left(\Sigma_{1,2}\right)$ is infinite non-abelian.

Let now $\Gamma \rightarrow H_{i}, i=1, \ldots, m$, be a collection of representations of the group $\Gamma$. The subgroup $H \subset \prod_{i=1}^{m} H_{i}$ is called $\Gamma$-diagonal, if there exists a partition $A_{1}, \ldots, A_{s}$ of $\{1,2, \ldots, m\}$ such that:

(1) All factors $H_{i}$, with $i \in A_{t}, 1 \leq t \leq s$ are equivalent as representations of $\Gamma$. Pick up some $i_{t} \in A_{t}$. Given some intertwining isomorphisms $L_{j, i_{t}}: H_{j} \rightarrow H_{i_{t}}, j \in A_{t} \backslash\left\{i_{t}\right\}$, we set:

$$
H_{A_{t}}=\left\{\left(x,\left(L_{j, i_{t}}(x)\right)_{j \in A_{t} \backslash\left\{i_{t}\right\}}\right), \quad x \in H_{i_{t}}\right\},
$$

which is the graph of the homomorphism $\oplus_{j \in A_{t} \backslash\left\{i_{t}\right\}} L_{j, i_{t}}$.

(2) Then there exist intertwining isomorphisms as above with the property that the group $H$ contains $\prod_{1 \leq t \leq s} H_{A_{t}}$. In particular, if all representations $H_{i}$ of $\Gamma$ are pairwise inequivalent, then $H=\prod_{i=1}^{m} H_{i}$.

We then have the following Hall lemma from [19]:

Lemma 3.1 ([19]). Let $\Gamma$ be a subgroup of the product $\prod_{i=1}^{m} H_{i}$ of the adjoint simple (i.e. connected, without center and whose Lie algebra is simple) Lie groups $H_{i}$. Assume that the projection of $\Gamma$ on each factor $H_{i}$ is Zariski dense. Then the Zariski closure of $\Gamma$ in $\prod_{i=1}^{m} H_{i}$ is a $\Gamma$-diagonal subgroup. 
We will use next the following classical result of Dieudonné ([4]) and Rickart ([34], Thm. 4.3):

Proposition 3.3 ([4, 34]). Any group isomorphism $L: U\left(W_{1}\right) \rightarrow U\left(W_{2}\right)$ between the unitary groups of Hermitian vectors spaces $W_{1}$ and $W_{2}$ has the form:

$$
L(h)=\chi(h) \cdot V^{-1} h V
$$

where the map $V: W_{1} \rightarrow W_{2}$ is either linear or anti-linear, and $\chi: U\left(W_{1}\right) \rightarrow U(1)$ is a homomorphism.

Lemma 3.2. Let $A$ and $B$ be primitive $2 p$-th roots of unity, for odd $p$. If $\left.\widetilde{\rho}_{p, A^{2},(p-3)}\right|_{\pi_{g}}$ and $\widetilde{\rho}_{p, B^{2},(p-3)}||_{\pi_{g}}$ are linearly or anti-linearly equivalent, then either $A=B$ or $A=\bar{B}$.

Proof. According to Proposition 3.3 the two representations in the same unitary group $U(W)$ are equivalent only if there exists an intertwiner (either linear or anti-linear) map $V: W \rightarrow W$ which conjugates the two representations, possibly up to twisting by a character $\chi: U(W) \rightarrow U(1)$. In our case the representations take values into the special unitary group and hence we can take $\chi=1$.

Observe that $V$ should send eigenspaces for $\widetilde{\rho}_{p, A^{2},(i)}(\gamma)$ to eigenspaces for $\widetilde{\rho}_{p, B^{2},(i)}(\gamma)$ of the same eigenvalues. If $\gamma$ a simple non-separating based loop on the surface, let $\gamma_{+}, \gamma_{-}$denote the curves obtained by slightly pushing left and right respectively. Then $\gamma_{+}, \gamma_{-}$and a small circle around the base point determine a pair of pants $S_{0,3}$ whose complement $S_{g-1,2}$ is a genus $g-1$ surface with two boundary components. Therefore

$$
\widetilde{\rho}_{p, A^{2},(i)}(\widetilde{\gamma}) x=A^{j(j+2)-k(k+2)} x \text {, if } x \in W_{0,(i, j, k)} \otimes W_{g-1,(j, k)}
$$

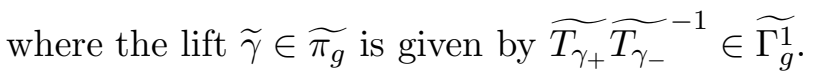

It follows that $V$ should send vector spaces of the form $W_{0,(i, j, k)} \otimes W_{g-1,(j, k)}$ into spaces of the same form associated to possibly different labels.

Consider $i=p-3$. Therefore, the only possibilities for $j, k$ such that $\operatorname{dim} W_{0,(i, j, k)}$ be non-zero is $j=p-3-2 m, k=2 m$, for some $2 m \in \mathcal{C}_{p}$. Now, observe that the symmetry exchanging the two boundary components induces an isomorphism between $W_{g-1,(j, k)}$ and $W_{g-1,(k, j)}$. Further, consider a circle embedded in $S_{g-1,2}$ which bounds a pair of pants along with the two boundary circles. If $\ell$ is a label for the third circle then the set of $p$-admissible $\ell$ for boundary labels $(j, k)$, where $j>k$ is strictly contained in the set of $p$-admissible values of $\ell$ for the boundary labels $(j-2, k+2)$. It follows that $\operatorname{dim} W_{g-1,(j, k)}$ are distinct for all values $j \geq k$, with $j+k=p-3$.

Therefore either $V$ keeps invariant each subspace $W_{0,(i, j, k)} \otimes W_{g-1,(j, k)}$ or else $V$ sends every $W_{0,(i, j, k)} \otimes W_{g-1,(j, k)}$ onto $W_{0,(i, k, j)} \otimes W_{g-1,(k, j)}$. Since the corresponding eigenvalues should be the same we derive that either $A=B$ or $A=\bar{B}$.

End of the proof of Theorem [3.1. The Hall Lemma 3.1] shows that the Zariski closure of $\rho_{p,(p-3)}\left(\pi_{g}\right)$ is all of $\mathbb{P} \mathbb{G}_{p,(p-3)}(\mathbb{R})$. Now, using $\left([19]\right.$, Lemma 3.6) we obtain that $\widetilde{\rho}_{p,(p-3)}\left(\widetilde{\pi}_{g}\right)$ is Zariski dense in $\mathbb{G}_{p,(p-3)}(\mathbb{R})$.

Finally notice that $\mathbb{G}_{g, p,(p-3)}$ contains $\mathbb{G}_{g-1, p}$ as a subgroup. In particular, for $g \geq 4$ each noncompact factor has rank at least 2, by [12]. We can follow the proof of this result in [12] for $i=0$ to obtain the result for $g=3$ as well. This proves the theorem.

3.2. Preliminaries on Verlinde formulas. We start by collecting a few properties of the dimensions of the space of conformal blocks. The main tool is the combinatorial description of the space of conformal blocks which admits a basis indexed by the set of $p$-admissible colorings of any uni-trivalent graph associated to the surface, possibly with colored boundary components, as explained in 2.1. As a consequence, if we split a surface $S_{g, k}$ by cutting along $r$ essential pairwise non isotopic simple curves into the subsurfaces $S_{h, s+r}$ and $S_{g-h-r+1, k-s+r}$ then we have a corresponding decomposition for the spaces of conformal blocks:

$$
W_{g, p,\left(i_{1}, \ldots, i_{k}\right)}=\sum_{j_{1}, \ldots j_{r} \in \mathcal{C}_{p}} W_{h, p,\left(i_{1}, \ldots, i_{s}, j_{1}, \ldots, j_{r}\right)} \otimes W_{g-h-r+1, p,\left(i_{s+1}, \ldots, i_{s}, j_{1}, \ldots, j_{r}\right)}
$$

Lemma 3.3.

$$
\operatorname{dim} W_{1, p,(j, i)}=\frac{(p-1-\max (i, j))(\min (i, j)+1)}{2}
$$


Proof. Direct computation using the combinatorial description of the vector space.

Lemma 3.4. For $k \in \mathcal{C}_{p}$ we have:

$$
\operatorname{dim} W_{2, p(k)}=\frac{1}{24} \cdot\left((k+1) p^{3}-\frac{3}{2} k(k+2) p^{2}+\frac{1}{2}\left(k^{3}+3 k^{2}-4\right) p\right)
$$

and, in particular:

$$
\operatorname{dim} W_{2, p,(p-3)}=\frac{p^{3}-p}{24}
$$

Proof. We have

$$
\operatorname{dim} W_{2, p(k)}=\sum_{j \in \mathcal{C}_{p}} \operatorname{dim} W_{1, p,(j)} \cdot \operatorname{dim} W_{1, p,(j, k)}
$$

then expand all terms using Lemma 3.3 .

\section{Lemma 3.5.}

$$
\operatorname{dim} W_{3, p,(p-3)}=\frac{1}{5760} \cdot p(p-1)(p-3)\left(7 p^{3}+28 p^{2}+101 p+80\right)+\frac{1}{24} \cdot\left(p^{3}-p\right)
$$

Proof. This is a consequence of Lemmas 3.3 and 3.4 along with

$$
\operatorname{dim} W_{3, p,(p-3)}=\sum_{j \in \mathcal{C}_{p}} \operatorname{dim} W_{2, p,(j)} \cdot \operatorname{dim} W_{1, p,(j, p-3)}
$$

Lemma 3.6. We have $\operatorname{dim} W_{g, p,(p-3)}>\operatorname{dim} W_{g, p,(0)}$, if $g \geq 3$.

Proof. We will prove by induction on $g$ that $\operatorname{dim} W_{g, p,(k)} \geq \operatorname{dim} W_{g, p,(0)}$, for any $k \in \mathcal{C}_{p}$, with equality only if $k=0, g \geq 3$ or $g=2$ and $k=p-3$.

When $g=2$ the explicit formula from Lemma 3.4 allows for a direct verification. Assume that our claim holds true for all genera up to $g$. We can write from Lemma 3.3

$$
\operatorname{dim} W_{g+1, p,(k)}=\sum_{j \in \mathcal{C}_{p}} \operatorname{dim} W_{g, p,(j)} \cdot \frac{(p-1-\max (k, j))(\min (k, j)+1)}{2}
$$

to be compared with

$$
\operatorname{dim} W_{g+1, p,(0)}=\sum_{j \in \mathcal{C}_{p}} \operatorname{dim} W_{g, p,(0)} \cdot \frac{(p-1-j)}{2}
$$

Now the induction hypothesis $\operatorname{dim} W_{g+1, p,(0)} \leq \operatorname{dim} W_{g, p,(j)}$ for all $j \in \mathcal{C}_{p}$ implies the claim for $g+1$.

Lemma 3.7. For any $g \geq 3, p \geq 7$ we have

$$
\operatorname{dim} W_{g+1, p,(p-3)}<\frac{\operatorname{dim} W_{g, p,(p-3)}\left(\operatorname{dim} W_{g,(p-3)}-1\right)}{2}
$$

Further, for $g=2$ we have the weaker inequality:

$$
\operatorname{dim} W_{3, p,(p-3)}<\left(\operatorname{dim} W_{2, p,(p-3)}\right)^{2}
$$

Proof. Recall the Verlinde formula (see [16]) computing the dimension of the space of conformal blocks:

$$
\operatorname{dim} W_{g, p,(k)}=\left(\frac{p}{4}\right)^{g-1} \sum_{s=1}^{\frac{p-1}{2}} \sin \left(\frac{(k+1) \pi s}{p}\right) \sin \left(\frac{\pi s}{p}\right)^{1-2 g}
$$

Set $\alpha_{s}=\left(\frac{p}{4}\right) \sin \left(\frac{\pi s}{p}\right)$. If $g \geq 4$ we have the following inequalities:

$$
\sum_{s} \alpha_{s}^{g} \leq \sum_{s} \alpha_{s}^{4(g-1) / 3}<\left(\sum_{s} \alpha_{s}\right)^{4 / 3}
$$

which imply that:

$$
\operatorname{dim} W_{g+1, p,(0)}<\left(\operatorname{dim} W_{g, p,(0)}\right)^{4 / 3}, \text { whenever } g \geq 4
$$


We derive from Lemma 3.6 that whenever $g \geq 4$ we have:

$$
\operatorname{dim} W_{g+1, p,(p-3)}<\operatorname{dim} W_{g+2, p,(0)} \leq\left(\operatorname{dim} W_{g+1, p,(0)}\right)^{4 / 3}<\left(\operatorname{dim} W_{g, p,(0)}\right)^{16 / 9}<\left(\operatorname{dim} W_{g, p,(p-3)}\right)^{16 / 9}
$$

On the other hand

$$
\left(\operatorname{dim} W_{g, p,(p-3)}\right)^{16 / 9}<\frac{\operatorname{dim} W_{g, p,(p-3)}\left(\operatorname{dim} W_{g, p,(p-3)}-1\right)}{2}
$$

if $g \geq 4$ and $p \geq 5$, since $\operatorname{dim} W_{g, p,(p-3)} \geq \operatorname{dim} W_{4,5,(2)}=75$.

Eventually, we have to check the case when $g=3$. From Lemma 3.4

$$
\operatorname{dim} W_{2, p,(k)}<\frac{1}{24}\left((k+1) p^{3}+\frac{k^{3}+3 k^{2}}{2} p\right)<\frac{p^{3}(3 p+5)}{48}
$$

We have the following crude upper bound:

$$
\sum_{j \in \mathcal{C}_{p}} \frac{(p-1-\max (k, j))(\min (k, j)+1)}{2}<\frac{1}{4} p^{3}
$$

which leads to the upper bounds:

$$
\operatorname{dim} W_{3, p,(k)}<\frac{p^{3}(3 p+5)}{48} \sum_{j \in \mathcal{C}_{p}} \frac{(p-1-\max (k, j))(\min (k, j)+1)}{2}<\frac{p^{6}(3 p+5)}{192}
$$

and further

$$
\operatorname{dim} W_{4, p,(k)}<\frac{p^{6}(3 p+5)}{192} \sum_{j \in \mathcal{C}_{p}} \frac{(p-1-\max (k, j))(\min (k, j)+1)}{2}<\frac{p^{9}(3 p+5)}{728}
$$

Now, if $p>35$ we have that

$$
\operatorname{dim} W_{4, p,(p-3)}<\frac{p^{9}(3 p+5)}{728}<\frac{7 p^{12}}{32 \times(5760)^{2}}<\frac{\operatorname{dim} W_{3, p,(p-3)}\left(\operatorname{dim} W_{3, p,(p-3)}-1\right)}{2}
$$

The cases when $5 \leq p \leq 35$ can be verified by a direct computer search.

Finally, the inequality claimed for $g=2$ is a consequence of Lemmas 3.4 and 3.5 .

Note that the inequality for $g \geq 3$ is actually valid with the same proof for all labels $i$ on the boundary circle.

Remark 3.1. The inequality stated in Lemma 3.7 for $g \geq 3$ does not hold when $g=2$. Indeed we have the following asymptotical behavior, derived from Lemma 3.5.

$$
\lim _{p \rightarrow \infty} \frac{\operatorname{dim} W_{3, p,(p-3)}}{\left(\operatorname{dim} W_{2, p,(p-3)}\right)^{2}} \simeq 0.7
$$

Lemma 3.8. There exist only finitely many $p$ such that $1+8 \operatorname{dim} W_{3, p,(p-3)}$ is a perfect square.

Proof. The function $f(p)=1+8 \operatorname{dim} W_{3, p,(p-3)}$ is a degree 6 square free polynomial in $p$. Faltings (see [7]) proved that a non-singular algebraic curve of genus at least two which is defined over a number field has only finitely many rational points (Mordell's conjecture). Since the projective curve given by the affine equation $y^{2}=f(x)$ is a hyperelliptic curve of genus 2 , the equation $y^{2}=f(x)$ has therefore only finitely many solutions in $\mathbb{Q}$.

Alternatively, we can use a classical theorem of Siegel, which asserts that a smooth affine algebraic curve defined over $\mathbb{Q}$ of genus at least one has only finitely many points with integral coordinates (see [20], chap. VI). In particular, a polynomial with integer coefficients and at least 3 distinct roots takes only finitely many square values on the integers. Although $(24)^{2}\left(1+8 \operatorname{dim} W_{3, p,(p-3)}\right)$ has rational coefficients, by considering the change of variable $p=5 q+s$, for each $s \in\{0,1,2,3,4\}$ we obtain five polynomials with integer coefficients to each of which Siegel's theorem applies. 
3.3. Proof of Proposition 3.1. Larsen and Wang in [21] proved the topological density of the image of the mapping class group of a closed surface of genus $g$. This result corresponds to the case when $i=0$ and $\zeta=A_{p}^{2}$. We will show that their proof suitably amended actually works for $i=p-3$ and $g \geq 2$. Some of the steps below are valid for every color $i$, but for the sake of simplicity we stick to $i=p-3$. In this section $p$ is an odd prime, $p \geq 5$.

The start point is the irreducibility of $\widetilde{\rho}_{g, p, \zeta,(i)}$, for any $i$, according to (the proof given by) Roberts ([35], see also [16], Cor. 3.2).

Consider the topological closure $\mathcal{G}_{g, p,(i)}$ of $\widetilde{\rho}_{p, A_{p}^{2},(i)}\left(\widetilde{\Gamma_{g, 1}}\right)$. We know from [10, that when $g \geq 2$, $p \geq 7$ the group $\mathcal{G}_{g, p,(i)}$ is infinite and non-abelian. Denote by $V_{g, p,(i)}$ the representation of $\mathcal{G}_{g, p,(i)}$ into $\mathbb{U}_{g, p, \zeta,(i)}$.

If the representation $V_{g, p,(i)}$ were self-dual, its restriction to $\widetilde{\Gamma_{g-1,1} \times} \Gamma_{1,1}$ would be a direct sum of self-dual and pairs of dual representations. The invariant subspace $W_{g-1,(0)} \otimes W_{1,(0, i)}$ is not selfdual (see [21, step 10), as $W_{g-1,(0)}$ is not self-dual. Moreover, it is not dual to $W_{g-1,(j)} \otimes W_{1,(j, i)}$, for any other values of $j$, since these subspaces are tensor products of irreducible representations and the corresponding dimensions of $W_{1,(j, i)}$ do not agree with that for $j=0$ unless $j=0$.

We wish now to prove our claim by induction on $g$. When $g=2$ we choose $i=p-3$. From above it follows that $\operatorname{dim} W_{2, p,(p-3)}=\frac{p^{3}-p}{24}$. Now the results from ([21], section 4) show that $\rho_{p, A_{p}^{2},(p-3)}\left(\Gamma_{g}^{1}\right)$ is topologically dense into $P \mathbb{U}_{2, p,(p-3)}$. We can show using the same lines that the result holds for large enough $p$, for any $i$.

Further it follows from [21] that:

(1) the restriction of $V_{g, p,(p-3)}$ to the identity component $\mathcal{G}_{g, p,(p-3)}^{\circ}$ of $\mathcal{G}_{g, p,(p-3)}$ is isotypic.

(2) For any normal subgroup $H \subset \mathcal{G}_{g, p,(p-3)}$ with the property that all morphisms $S L_{2}(\mathbb{Z} / p \mathbb{Z}) \rightarrow$ $\mathcal{G}_{g, p,(p-3)} / H$ are trivial, the representation of $H$ into $V_{g, p,(p-3)}$ is tensor indecomposable.

(3) Moreover, $V_{g, p,(p-3)}$ is irreducible as a $\mathcal{G}_{g, p,(p-3)^{-}}^{\circ}$-representation.

Further the content of Lemma 3.7 is the extension of ([21], Lemma 12) to the case of surfaces with boundary. This is the main condition needed to prove the induction step from $g$ to $g+1$. It actually works for all $g \geq 3$, except for $g=2$.

When $g=2$ we only obtain that $\mathcal{G}_{3, p,(p-3)}^{\circ}$ is a simple compact Lie group of type $A_{n}$ and the representation $V_{3, p,(p-3)}$ is either the standard one or else the exterior or the symmetric square. In particular, if this representation were not the standard one, then $\operatorname{dim} W_{3, p,(p-3)}$ would be of the form $m(m+1) / 2$, for some natural number $m \in\{n, n+1\}$. This situation could only occur for finitely many $p$, according to Lemma 3.8 .

Eventually the arguments from ([21], steps 14 and 15) show that the identity component $\mathcal{G}_{g, p,(p-3)}^{\circ}$ is a simple compact Lie group and for $g \geq 3, p \geq 7$ we have the equality $\mathcal{G}_{g, p,(p-3)}=S \mathbb{U}_{g, p,(p-3)}$. Thus $\widetilde{\rho}_{p, A_{p}^{2},(p-3)}\left(\widetilde{\Gamma_{g, 1}}\right)$ is topologically dense into $S \mathbb{U}_{g, p,(p-3)}$. This implies that $\widetilde{\rho}_{p, \zeta,(p-3)}\left(\widetilde{\Gamma_{g}^{1}}\right)$ is Zariski dense into $S \mathbb{U}_{g, p, \zeta,(p-3)}$ for all primitive roots of unity $\zeta$.

3.4. Trace fields. Recall that $S \mathbb{U}_{g, p,(i)}$ is an absolutely almost simple simply connected algebraic group defined over $\mathbb{Q}\left(\zeta_{p}+\overline{\zeta_{p}}\right)$ (i.e. its proper normal algebraic subgroups are finite). The adjoint trace field of a subgroup $\Delta \subset S \mathbb{U}_{g, p,(i)}$ is the field $\mathbb{Q}(\operatorname{tr}(A d(x)), x \in \Delta)$, where $A d$ is the adjoint representation of $S \mathbb{U}_{g, p,(i)}$. We have the following extension of the corresponding result for mapping class groups of closed surfaces from ([28], section 4.3):

Lemma 3.9. Up to rescaling $\rho_{p,(p-3)}$ by some $2 p$-th root of unity we can insure that the adjoint trace field of $\widetilde{\rho}_{p,(p-3)}\left(\widetilde{\pi_{g}}\right)$ is $\mathbb{Q}\left(\zeta_{p}+\overline{\zeta_{p}}\right)$.

Proof. If $\ell$ denotes the adjoint trace field in the statement then $\ell \subset \mathbb{Q}\left(\zeta_{p}+\overline{\zeta_{p}}\right)$. The Zariski density and classical theorems of Vinberg (see [28], Prop.4.2) show that $S \mathbb{U}_{g, p,(p-3)}$ is defined over $\ell$ and $A d\left(\widetilde{\rho}_{p,(p-3)}\left(\widetilde{\pi_{g}}\right)\right)$ is contained in the group $A d\left(S \mathbb{U}_{g, p,(p-3)}\right)(\ell)$ of $\ell$ points of the adjoint group $A d\left(S \mathbb{U}_{g, p,(p-3)}\right)$. If we show that $\left.\widetilde{\rho}_{p,(p-3)}\left(\widetilde{\pi_{g}}\right)\right)$ is contained in the group $S \mathbb{U}_{g, p,(p-3)}(\ell)$ then the argument of $\left([28\right.$, section 4.3$)$ will imply that $\ell=\mathbb{Q}\left(\zeta_{p}+\overline{\zeta_{p}}\right)$.

If $Z$ is the center of $S \mathbb{U}_{g, p,(p-3)}$, then we have an exact sequence

$$
Z\left(\mathbb{Q}\left(\zeta_{p}+\overline{\zeta_{p}}\right)\right) \rightarrow S \mathbb{U}_{g, p,(p-3)}\left(\mathbb{Q}\left(\zeta_{p}+\overline{\zeta_{p}}\right)\right) \rightarrow A d\left(S \mathbb{U}_{g, p,(p-3)}\right)\left(\mathbb{Q}\left(\zeta_{p}+\overline{\zeta_{p}}\right)\right)
$$


Let $\sigma \in \operatorname{Gal}\left(\mathbb{Q}\left(\zeta_{p}+\overline{\zeta_{p}}\right) / \ell\right)$. Then we have a homomorphism

$$
\begin{gathered}
f: \widetilde{\rho}_{p,(p-3)}\left(\widetilde{\pi_{g}}\right) \rightarrow Z^{1}\left(\operatorname{Gal}\left(\mathbb{Q}\left(\zeta_{p}+\overline{\zeta_{p}}\right) / \ell\right), Z\left(\mathbb{Q}\left(\zeta_{p}+\overline{\zeta_{p}}\right)\right)\right. \\
f(\gamma)(\sigma)=\gamma \sigma\left(\gamma^{-1}\right), \text { for } \gamma \in \widetilde{\rho}_{p,(p-3)}\left(\widetilde{\pi_{g}}\right), \sigma \in \operatorname{Gal}\left(\mathbb{Q}\left(\zeta_{p}+\overline{\zeta_{p}}\right) / \ell\right)
\end{gathered}
$$

The group of 1-cocycles $Z^{1}$ is an abelian group and hence $f$ factors through the abelianization $H_{1}\left(\widetilde{\rho}_{p,(p-3)}\left(\widetilde{\pi_{g}}\right)\right.$ which is a quotient of $(\mathbb{Z} / p \mathbb{Z})^{2 g}$. On the other hand the group cohomology $H^{1}(H, Z)$ is killed by the order of the finite group $H$. Now, the order of the group $\operatorname{Gal}\left(\mathbb{Q}\left(\zeta_{p}+\overline{\zeta_{p}}\right) / \ell\right)$ is a divisor of $\frac{p-1}{2}$. Thus elements in the image of the map induced by $f$ :

$$
f_{*}: \widetilde{\rho}_{p,(p-3)}\left(\widetilde{\pi_{g}}\right) \rightarrow H^{1}\left(\operatorname{Gal}\left(\mathbb{Q}\left(\zeta_{p}+\overline{\zeta_{p}}\right) / \ell\right), Z\left(\mathbb{Q}\left(\zeta_{p}+\overline{\zeta_{p}}\right)\right)\right.
$$

should be killed by both $p$ and some divisor of $\frac{p-1}{2}$ and hence they are trivial in cohomology. Therefore there exists $a$ in the center $Z\left(\mathbb{Q}\left(\zeta_{p}+\overline{\zeta_{p}}\right)\right)$ such that

$$
f(\gamma)(\sigma)=a \cdot \sigma\left(a^{-1}\right)
$$

and thus rescaling $\rho_{p,(p-3)}$ by a will insure that $f(\gamma)$ is trivial for every $\gamma$ and hence $\left.\widetilde{\rho}_{p,(p-3)}\left(\widetilde{\pi_{g}}\right)\right)$ is contained in the group $S \mathbb{U}_{g, p,(p-3)}(\ell)$. Note now that the center $Z\left(\mathbb{Q}\left(\zeta_{p}+\overline{\zeta_{p}}\right)\right)$ consists of scalars which are roots of unity in $\mathbb{Q}\left(\zeta_{p}+\overline{\zeta_{p}}\right)$, and thus they are $2 p$-th roots of unity.

\section{Proofs of the Main theorems}

4.1. Abundance of finite quotients. We will need the following versions of the strong approximation theorem due to Nori-Weisfeiler. First, we record the statement due to Nori for algebraic groups defined over $\mathbb{Q}$ :

Theorem 4.1 ([30], Thm.5.4). Let $G$ be a connected linear algebraic group $G$ defined over $\mathbb{Q}$ and $\Lambda \subset G(\mathbb{Z})$ be a Zariski dense subgroup. Assume that $G(\mathbb{C})$ is simply connected. Then the completion of $\Lambda$ with respect to the congruence topology induced from $G(\mathbb{Z})$ is an open subgroup in the group $G(\widehat{\mathbb{Z}})$ of points of $G$ over the pro-finite completion $\widehat{\mathbb{Z}}$ of $\mathbb{Z}$.

Further, in ([28], Thm. 2.6) Masbaum and Reid stated the following consequence of the approximation theorem stated by Weisfeiler ([4], Thm. 10.5, Cor. 10.6), which is now valid for algebraic groups defined over number fields:

Theorem 4.2 ([28], Thm.2.6). If $\Delta \subset S \mathbb{U}_{g, p,(i)}(\mathbb{Q}(\zeta+\bar{\zeta}))$ is a Zariski dense subgroup of $S \mathbb{U}_{g, p,(i)}$ such that the adjoint trace field of $\Delta$ is $\mathbb{Q}(\zeta+\bar{\zeta})$, then for all but finitely many primes $\mathfrak{p}$ in $\mathbb{Q}(\zeta+\bar{\zeta})$ the reduction homomorphism $\Delta \rightarrow S \mathbb{U}_{g, p,(i)}\left(\mathbf{F}_{\mathfrak{p}}\right)$ is surjective, where $\mathbf{F}_{\mathfrak{p}}$ denotes the residue field $\mathbb{Q}(\zeta+\bar{\zeta}) / \mathfrak{p}$.

Our key ingredient in the proofs of the main theorems is the following result showing that infinitely many finite groups of Lie type should occur among the quotients of a Burnside-type group:

Proposition 4.1. Let $g \geq 2$ and $p \equiv 3(\bmod 4)$, p large enough prime. Then, for all but finitely many primes $q$ there exist surjective homomorphisms $B\left(\widetilde{\pi_{g}}, p, \mathcal{S}\left(S_{g}\right)\right) \rightarrow \mathbb{G}_{g, p,(p-3)}\left(\mathbb{Z} / q^{k} \mathbb{Z}\right)$ and $B\left(\pi_{g}, p, \mathcal{S}\left(S_{g}\right)\right) \rightarrow \mathbb{P} G_{g, p,(p-3)}\left(\mathbb{Z} / q^{k} \mathbb{Z}\right)$. Moreover, for infinitely many $q$ the finite groups on the right hand side surject onto $P S L\left(N_{g, p}, \mathbf{F}_{q}\right)$, where $\mathbf{F}_{q}$ denotes the finite field on $q$ elements and $N_{g, p}=\operatorname{dim} W_{g, p,(p-3)}$.

Proof. The linear algebraic group $G=\mathbb{G}_{g, p,(p-3)}$ satisfies the assumptions of Nori's Theorem 4.1 , If we take $\Lambda$ to be a finite index subgroup of $\widetilde{\rho}_{p,(p-3)}\left(\widetilde{\pi_{g}}\right)$, then Theorem 4.1 implies our claim for $k=1$.

In fact $\widetilde{\rho}_{p,(p-3)}||_{\pi_{g}}$ factors through $B\left(\widetilde{\pi_{g}}, p, \mathcal{S}\left(S_{g}\right)\right)$, since each homotopy class of a simple closed curve on $S_{g}$ is sent into the composition of two commuting Dehn twists in $\widetilde{\Gamma_{g}^{1}}$. Moreover, the Dehn twist along the boundary curve $c=\partial S_{g}$ is central in $\Gamma_{g, 1}$, and hence the image by $\widetilde{\rho}_{g, p}$ of the center of $\widetilde{\Gamma_{g}^{1}}$ consists of central elements of finite order $p$.

Then a classical result due to Serre (see [38]) for $G L(2)$ and extended by Vasiu (see [42]) to all reductive linear algebraic groups defined over $\mathbb{Q}$ improves the surjectivity statement to all $k \geq 1$. 
An alternate approach for $k=1$ would be to use directly the Nori-Weisfeiler approximation theorem on $S \mathbb{U}_{g, p,(p-3)}$. From Lemma 3.9 the group $\widetilde{\rho}_{p, A_{p}^{2},(p-3)}\left(\widetilde{\pi_{g}}\right) \subset S \mathbb{U}_{g, p,(p-3)}$ has trace field $\mathbb{Q}(\zeta+\bar{\zeta})$, up to possibly translating it by a root of unity. Therefore, by Theorem 4.2 for all but finitely many primes $\mathfrak{p}$ in the trace field the reduction mod $\mathfrak{p}$ is well-defined and provides a surjection $\widetilde{\rho}_{p, A_{p}^{2},(p-3)}\left(\widetilde{\pi_{g}}\right) \rightarrow S \mathbb{U}_{g, p,(p-3)}\left(\mathbf{F}_{\mathfrak{p}}\right)$. According to the discussion in ([40], p.55; [31], 2.3.3) the group $S \mathbb{U}_{g, p,(p-3)}\left(\mathbf{F}_{\mathfrak{p}}\right)$ is either a special unitary group, when $\mathfrak{p}$ is prime or ramified in $\mathbb{Q}(\zeta)$ or else a special linear group, when $\mathfrak{p}$ splits completely in $\mathbb{Q}(\zeta)$. In particular, if $q$ is a rational prime which splits completely in $\mathbb{Q}(\zeta)$ and $\mathfrak{p}$ a prime in $\mathbb{Q}(\zeta+\bar{\zeta})$ which divides $q$, then $S \mathbb{U}_{g, p,(p-3)}\left(\mathbf{F}_{\mathfrak{p}}\right)$ is isomorphic to $S L\left(N_{g, p}, \mathbf{F}_{q}\right)$, for all but finitely many $\mathfrak{p}$.

We now record the following version of Hall's lemma (see [17]) for finite groups, due to Dunfield and Thurston:

Lemma 4.1 ([6], Lemma 3.7). Suppose that we have a set of epimorphisms $f_{i}: G \rightarrow H_{i}$, where $H_{1}, H_{2}, \ldots, H_{k}$ are non-abelian simple groups. If $f_{i}$ are pairwise non-equivalent, namely there is no isomorphism between $\alpha: H_{i} \rightarrow H_{j}$ such that $\alpha \circ f_{i}=f_{j}$, for $i \neq j$, then the map

$$
\left(f_{1}, f_{2}, \ldots, f_{k}\right): G \rightarrow H_{1} \times H_{2} \times \cdots \times H_{k}
$$

is surjective.

Proposition 4.2. For large enough prime $p \equiv 3(\bmod 4)$ and $g \geq 2$ the group $B\left(\pi_{g}, \widehat{p, \mathcal{S}}\left(S_{g}\right)\right)$ is neither finite-by-solvable, nor solvable-by-finite.

Proof. For large enough prime $q$ the surjective maps $B\left(\pi_{g}, p, \mathcal{S}\left(S_{g}\right)\right) \rightarrow \mathbb{P} G_{p,(p-3)}\left(\mathbb{Z} / q^{k} \mathbb{Z}\right)$ induce a continuous surjective homomorphism: $B\left(\widehat{\pi_{g}, \widehat{p, \mathcal{S}}}\left(S_{g}\right)\right) \rightarrow P \mathbb{G}_{p,(p-3)}\left(\mathbb{Z}_{q}\right)$.

If $B\left(\pi_{g}, \widehat{p, \mathcal{S}}\left(S_{g}\right)\right)$ had a prosolvable normal subgroup of finite index at most $N$, then $P \mathbb{G}_{g, p,(p-3)}\left(\mathbb{Z}_{q}\right)$ would also have a prosolvable normal subgroup of index at most $N$. But the index of the largest normal prosolvable group within $P \mathbb{G}_{g, p,(p-3)}\left(\mathbb{Z}_{q}\right)$ goes to infinity with $q$. It is well-known that $P \mathbb{G}_{g, p,(p-3)}(\mathbb{Z} / q \mathbb{Z})$ are finite simple groups ([40], p.55; 31, 2.3.3). More precisely, by Proposition 4.1 we can find infinitely many finite groups of the form $P S L\left(N_{g, p}, \mathbf{F}_{q}\right)$ and $P U\left(N_{g, p}, \mathbf{F}_{q}\right)$ among these quotients. In particular, a normal solvable subgroup of $P \mathbb{G}_{g, p,(p-3)}\left(\mathbb{Z}_{q}\right)$ must project to the trivial subgroup of $P \mathbb{G}_{g, p,(p-3)}(\mathbb{Z} / q \mathbb{Z})$ and hence has index at least the size of the later. This is optimal, as

$$
P \mathbb{G}_{g, p,(p-3)}\left(q \mathbb{Z}_{q}\right)=\operatorname{ker}\left(P \mathbb{G}_{g, p,(p-3)}\left(\mathbb{Z}_{q}\right) \rightarrow P \mathbb{G}_{g, p,(p-3)}(\mathbb{Z} / q \mathbb{Z})\right)
$$

is a pro- $q$ group and hence it is prosolvable. Now the size of $P \mathbb{G}_{g, p,(p-3)}(\mathbb{Z} / q \mathbb{Z})$ goes to infinity with $q$. Therefore $B\left(\widehat{\pi_{g}, p,} M\right)$ is not virtually prosolvable.

An alternate proof is as follows. Since $P \mathbb{G}_{g, p,(p-3)}(\mathbb{Z} / q \mathbb{Z})$ are simple non-abelian groups, for large $q$ they are pairwise non-isomorphic. From Hall's Lemma 4.1 we derive that the product homomorphism

$$
B\left(\pi_{g} \widehat{, p, \mathcal{S}}\left(S_{g}\right)\right) \rightarrow \oplus_{q \geq m(p)} P \mathbb{G}_{g, p,(p-3)}(\mathbb{Z} / q \mathbb{Z})
$$

is surjective.

According to ([33], Corollary 4.2.4) a prosolvable group has all its finite quotients solvable. In our case any finite index normal subgroup of $B\left(\pi_{g}, \widehat{p, \mathcal{S}}\left(S_{g}\right)\right)$ surjects onto infinitely many simple groups, and hence it cannot be virtually prosolvable.

Eventually, suppose that $B\left(\pi_{g}, \widehat{p, \mathcal{S}}\left(S_{g}\right)\right)$ is solvable-by-finite, namely it contains a finite normal subgroup $L$ such that the quotient $B\left(\pi_{g}, \widehat{p, \mathcal{S}}\left(S_{g}\right)\right) / L$ is prosolvable. Then the image of $L$ in every large enough finite simple quotient $P \mathbb{G}_{g, p,(p-3)}(\mathbb{Z} / q \mathbb{Z})$ should be trivial, as it cannot be the whole group by cardinality reasons. Therefore, $B\left(\pi_{g}, \widehat{p, \mathcal{S}}\left(S_{g}\right)\right) / L$ surjects onto infinitely many finite simple groups $P \mathbb{G}_{g, p,(p-3)}(\mathbb{Z} / q \mathbb{Z})$. By the arguments above this contradicts the fact that $B\left(\pi_{g} \widehat{, p, \mathcal{S}}\left(S_{g}\right)\right) / L$ was supposed (virtually) prosolvable. 
4.2. Proof of Theorem 1.1. The case $m=1$ is settled in Proposition 4.2 above.

Now, in order to prove a similar statement for $B\left(\pi_{g}, p, \mathcal{S}_{m}\left(S_{g}\right)\right)$, where $m \geq 2$ we have to pass to a finite cover of $S_{g}$. Indeed the classes of closed immersed based loops in $S_{g}$ with no more than $m$ self-intersections up to a homeomorphism of $S_{g}$ form a finite set. Choose a set of based loops $M$ of representatives of this set.

According to a classical Theorem of Scott ([36, 37]), given a based loop $\gamma$ on $S_{g}$ there exists a finite cover $S_{h}$ of $S_{g}$ and some $d$ such that the loop $\gamma^{d}$ lifts to an embedded loop in $S_{h}$. There exists then a finite characteristic cover, say of degree $d$, of pointed surfaces $f:\left(S_{h}, \tilde{z}\right) \rightarrow\left(S_{g}, z\right)$ so that the $d$-th powers of all based loops from $M$ admit simple lifts based at $\tilde{z}$. It follows that the $d$-th powers of based loops from $\mathcal{S}_{n}\left(S_{g}\right)$ lift to simple based loops in $S_{h}$.

Observe that the restriction of any automorphism of $\pi_{g}$ to the (image of) $\pi_{h}$, viewed as a subgroup, is an automorphism of $\pi_{h}$. This defines a homomorphism $F: \Gamma_{g}^{1} \rightarrow \Gamma_{h}^{1}$. If $\varphi \in \Gamma_{g}^{1}$ is such that $\varphi(x)=x$, for any $x \in \pi_{h}$, then $\varphi\left(x^{d}\right)=x^{d}$, for any $x \in \pi_{g}$. Since surface groups are bi-orderable (this goes back to Magnus) we have $\varphi(x)=x$ for any $x \in \pi_{g}$, as a strict inequality for some $x$ would imply a strict inequality for its $d$-th powers. Therefore $F$ is injective.

Recall that for any based loop $\gamma$ on $S_{g}$ we have $f\left(f^{-1}(\gamma)\right)=\gamma^{d} \in \pi_{1}\left(S_{g}, z\right)$, as the loop $\gamma$ is traveled $d$-times. If $\gamma \in \mathcal{S}_{m}\left(S_{g}\right)$, there exists some simple lift $\widetilde{\gamma}$ based at $\tilde{z}$. It follows that $f(\widetilde{\gamma})=\gamma^{m(\gamma)} \in \pi_{1}\left(S_{g}, z\right)$, where $m(\gamma)$ is a divisor of $d$.

Denote by $a d_{S_{g}, \gamma}$ the action by conjugacy by $\gamma$, namely the image of $\gamma$ into $\Gamma_{g}^{1}=\operatorname{Aut}^{+}\left(\pi_{g}\right)$. As $\gamma^{d}$ belongs to the image of $\pi_{h}$ we can compute:

$$
F\left(a d_{S_{g}, \gamma^{d}}\right)=a d_{S_{h}, \widetilde{\gamma}^{d / m(\gamma)}}
$$

It follows that the image by $F$ of the group $\mathcal{S}_{m}\left(S_{g}\right)[n d]$ is contained into $\mathcal{S}\left(S_{h}\right)[n]$.

Although $F\left(\pi_{g}\right)$ is not contained into $\pi_{h}$, it contains $\pi_{h}$ of finite index dividing $d$ since for any element $\gamma \in \pi_{g}$ its image $F\left(a d_{S_{g}, \gamma}\right)^{d} \in \pi_{h}$.

Further the map $F$ induces a homomorphism

$$
\bar{F}: B\left(\pi_{g}, n d, \mathcal{S}_{m}\left(S_{g}\right)\right) \rightarrow \Gamma_{h}^{1} / F\left(\mathcal{S}_{m}\left(S_{g}\right)[n d]\right)
$$

Now, the subgroup $\pi_{h} / F\left(\mathcal{S}_{m}\left(S_{g}\right)[n d]\right)$ is of finite index into the image $\bar{F}\left(B\left(\pi_{g}, n d, \mathcal{S}_{m}\left(S_{g}\right)\right)\right)$. As

$$
F\left(\mathcal{S}_{m}\left(S_{g}\right)[n d]\right) \subset \mathcal{S}\left(S_{h}\right)[n] \subset \pi_{h}
$$

and $\pi_{h}$ is a normal subgroup in $\Gamma_{h}^{1}$, the group $\pi_{h} / F\left(\mathcal{S}_{m}\left(S_{g}\right)[n d]\right)$ surjects onto the Burnside-type group $B\left(\pi_{h}, n, \mathcal{S}\left(S_{h}\right)\right)$.

It follows that the group $B\left(\pi_{g}, n d, \mathcal{S}_{m}\left(S_{g}\right)\right)$ has a finite index subgroup which surjects onto $B\left(\pi_{h}, n, \mathcal{S}\left(S_{h}\right)\right)$, and in particular it is not virtually prosolvable nor solvable-by-finite or finite-bysolvable.

Remark 4.1. If we had proven that $d=1$ is convenient for all $m$ then the family of finite quotients of $B\left(\pi_{g}, n d, \mathcal{S}_{m}\left(S_{g}\right)\right)$ would provide a negative answer to Problem 4' from [45].

Remark 4.2. It would be interesting to know whether the image of $B\left(\widehat{\pi_{g}, p, M}\right) \rightarrow \prod_{q \geq m(p)} \mathbb{G}_{p,(i)}\left(\mathbb{Z}_{q}\right)$ is open.

Remark 4.3. The arithmetic group $\mathbb{G}_{p,(p-3)}(\mathbb{Z})$, for $g \geq 3$ and prime $p \geq 5$ has the congruence property. This follows from results of Tomanov (see [41], Main Thm. (a)) and Prasad and Rapinchuk (see [32, Thm. 2.(1) and Thm. 3) on the congruence kernel for $\mathbb{Q}$-anisotropic algebraic groups of type ${ }^{2} A_{n-1}$, with $n \geq 4$. Moreover, $\mathbb{G}_{p,(p-3)}(\mathbb{Z})$ is cocompact in $\mathbb{G}_{p,(p-3)}(\mathbb{R})$, since it is $\mathbb{Q}$-anisotropic, by a classical result of Borel and Harish-Chandra (see [3]).

4.3. Proof of Corollary 1.3. We have the following sequence of inclusions:

$$
\rho_{p,(p-3)}\left(\pi_{g}\right) \subset \rho_{p,(p-3)}(\Gamma) \subset \rho_{p,(p-3)}\left(\Gamma_{g}^{1}\right) \subset P \mathbb{G}_{g, p,(p-3)}(\mathbb{Z})
$$

Proposition 4.1 shows that for $g \geq 2$ and large enough prime $p \equiv 3(\bmod 4)$ the reduction $\bmod q^{k}$ sends $\rho_{p,(p-3)}\left(\pi_{g}\right)$ onto $P \mathbb{G}_{g, p,(p-3)}\left(\mathbb{Z} / q^{k} \mathbb{Z}\right)$, for all but finitely many primes $q$. Therefore, all groups in the sequence above have the same image $P \mathbb{G}_{g, p,(p-3)}\left(\mathbb{Z} / q^{k} \mathbb{Z}\right)$ under the reduction $\bmod q^{k}$. 
For infinitely many $q$ the groups $P \mathbb{G}_{g, p,(p-3)}(\mathbb{Z} / q \mathbb{Z})$ are either of the form $P S L\left(N_{g, p}, \mathbf{F}_{q}\right)$ or $P S U\left(N_{g, p}, \mathbf{F}_{q}\right)$, for some $N_{g, p}$ going to infinity with $p$. This gives the first assertion of Corollary 1.3 .

Eventually, the fundamental group $\pi_{1}\left(M^{3}\right)$ of the fibered 3-manifold $M^{3}$ with monodromy $\varphi \in$ Aut $^{+}\left(\pi_{g}\right)$ is isomorphic to the semi-direct product $\pi_{g} \rtimes_{\varphi} \mathbb{Z}$, where the action of the generator of $\mathbb{Z}$ on $\pi_{g}$ is given by $\varphi$. Now, $\pi_{1}\left(M^{3}\right)$ embedds in $\Gamma_{g}^{1}$, since it is isomorphic to the preimage of the group $\langle\bar{\varphi}\rangle \subset \Gamma_{g}$ generated by the class $\bar{\varphi} \in \Gamma_{g}$ of $\varphi$, under the homomorphism $\Gamma_{g}^{1} \rightarrow \Gamma_{g}$. Then the claim follows from above.

Remark 4.4. Quantum representations are asymptotically faithful (see [1, 9]); for a surface of genus $g \geq 2$ with one boundary component and an infinite set $A$ of odd numbers we have (see [9], Thm.3.3):

$$
\cap_{p \in A, i \in \mathcal{C}_{p}} \operatorname{ker} \rho_{g, p,(i)}=1 \in \Gamma_{g}^{1}
$$

It seems that ([25]) the methods of [23, 24] could improve the asymptotic faithfulness above to the case where we only consider a single boundary color for each $p$, namely that:

$$
\cap_{p \in A} \operatorname{ker} \rho_{g, p,(f(p))}=1 \in \Gamma_{g}^{1}, \text { provided that } \lim _{p \rightarrow \infty} \frac{f(p)}{p}=1
$$

Now, a non-trivial element of $P \mathbb{G}_{g, p,(p-3)}(\mathbb{Z})$ can be detected by reduction modulo some prime $q$ belonging to any given infinite set of primes. Thus projections onto finite simple quotients $P \mathbb{G}_{g, p,(p-3)}(\mathbb{Z} / q \mathbb{Z})$ could detect any non-trivial element of $\Gamma_{g}^{1}$, when $p$ and $q$ belong to (any) infinite sets of primes.

4.4. Proof of Theorem 1.4. Consider the homomorphism $\Psi_{g, p, q}$ obtained by composing the projection on a simple factor, the reduction $\bmod q^{k}$ and $\rho_{p,(p-3)}$ as follows:

$$
\Gamma_{g}^{1} \rightarrow \rho_{p,(p-3)}\left(\Gamma_{g}^{1}\right) \subset P \mathbb{G}_{g, p,(p-3)}(\mathbb{Z}) \rightarrow P \mathbb{G}_{g, p,(p-3)}(\mathbb{Z} / q \mathbb{Z})
$$

Recall from the first lines of the proof of Corollary 1.3 that for any large prime $p \equiv 3(\bmod 4)$ and large enough prime $q$ we have:

$$
\Psi_{g, p, q}\left(\pi_{g}\right)=\Psi_{g, p, q}\left(\Gamma_{g}^{1}\right)=P \mathbb{G}_{g, p,(p-3)}(\mathbb{Z} / q \mathbb{Z})
$$

We obtained therefore infinitely many surjective homomorphisms $\Psi: \mathrm{Aut}^{+}\left(\pi_{g}\right) \rightarrow F$ onto finite simple groups $F$ of Lie type, with the property that $\Psi\left(\pi_{g}\right)=F$ (we dropped the subscripts to simplify the notation). We now claim that $\left.\operatorname{ker} \Psi\right|_{\pi_{g}}$ are characteristic subgroups, which will settle the result.

Observe first that every subgroup ker $\left.\Psi\right|_{\pi_{g}} \subset \pi_{g}$ is $\operatorname{Aut}^{+}\left(\pi_{g}\right)$-invariant because:

$$
\left.\Psi\right|_{\pi_{g}}(\varphi(x))=\Psi\left(\varphi x \varphi^{-1}\right)=1 \text {, for }\left.x \in \operatorname{ker} \Psi\right|_{\pi_{g}}, \varphi \in \operatorname{Aut}^{+}\left(\pi_{g}\right)
$$

We are almost done since $\operatorname{Aut}^{+}\left(\pi_{g}\right)$ is an index 2 subgroup of the group of all automorphisms $\operatorname{Aut}\left(\pi_{g}\right)$. To proceed further, realize $S_{g}$ in the Euclidean space as the double along the boundary of $S_{\frac{g}{2}, 1}$, if $g$ is even and $S_{\frac{g-1}{2}, 2}$, when $g$ is odd, respectively. Let $\tau$ denote the Euclidean symmetry exchanging the two halves of $S_{g}$, so that $\tau$ is an involution reversing the orientation of $S_{g}$. We denote by the same letter the corresponding mapping class $\tau \in \operatorname{Aut}\left(\pi_{g}\right)$. Further, $\operatorname{Aut}\left(\pi_{g}\right)$ is generated by $\operatorname{Aut}^{+}\left(\pi_{g}\right)$ and an arbitrary orientation reversing mapping class, in particular $\tau$.

We have now the following lemma whose proof is postponed a few lines:

Lemma 4.2. The homeomorphism $\tau$ induces an anti-linear map $\tau_{*}: W_{g, p,(i)} \rightarrow W_{g, p,(i)}$ which coincides with the anti-linear involution $J$ induced by the complex conjugation of coordinates.

The group of homeomorphisms of $\Sigma_{g, 1}$ which are identity on the boundary acts on the space of conformal blocks $W_{g, p,(i)}$, since their construction is functorial. This action provides a representation of $\operatorname{Aut}\left(\pi_{g}\right)$ into the general linear group of the real vector space underlying $W_{g, p,(i)}$ which extends $\rho_{p, \zeta,(i)}$. We keep the same notation $\rho_{p, \zeta,(i)}$ for this extension.

Now, Lemma 4.2 gives us:

$$
\rho_{p, \zeta,(i)}\left(\tau x \tau^{-1}\right)=J \rho_{p, \zeta,(i)}(x) J=\rho_{p, \bar{\zeta},(i)}(x), \text { for } x \in \operatorname{Aut}^{+}\left(\pi_{g}\right)
$$


Further, if we identify $\tau(x) \in \pi_{g}$ with its image by the point pushing map $\pi_{g} \rightarrow \Gamma_{g}^{1}$ arising in the Birman exact sequence, we can write:

$$
\tau(x)=\tau x \tau^{-1}, \text { for } x \in \pi_{g}
$$

We then obtain from above:

$$
\rho_{p, \zeta,(i)}(\tau(x))=\rho_{p, \zeta,(i)}\left(\tau x \tau^{-1}\right)=J \rho_{p, \zeta,(i)}(x) J=\rho_{p, \bar{\zeta},(i)}(x), \text { for } x \in \pi_{g}
$$

Therefore, $\operatorname{ker}\left(\left.\rho_{p,(i)}\right|_{\pi_{g}}\right)$ is $\tau$-invariant and hence a characteristic subgroup of $\pi_{g}$.

The complex conjugation $J$ induces an automorphism of $\mathbb{G}_{g, p,(i)}$ keeping invariant the lattice $\mathbb{G}_{g, p,(i)}(\mathbb{Z})$. Then the two surjective homomorphisms $\left.\Psi\right|_{\pi_{g}}$ and $\left.\Psi\right|_{\pi_{g}} \circ \tau: \pi_{g} \rightarrow F$ are equivalent, so that $\operatorname{ker}\left(\left.\Psi\right|_{\pi_{g}}\right)$ is both $\operatorname{Aut}^{+}\left(\pi_{g}\right)$-invariant and $\tau$-invariant and hence a characteristic subgroup, as claimed. This proves Theorem 1.4 .

Proof of Lemma 4.2. The homeomorphism $\tau$ extends to $S^{3}$ and sends any link into its mirror image. Recall that the Kauffman bracket invariant \langle\rangle$_{A}$ at the parameter $A$ is well-behaved with respect to the mirror symmetry, namely we have:

$$
\langle\tau(K)\rangle_{A}=\langle K\rangle_{A^{-1}}
$$

Therefore $\tau$ induces a map at the level of skein modules of the handlebodies $H_{g}$ (and $\bar{H}_{g}$ ) still denoted $\tau: S_{A}\left(H_{g}\right) \rightarrow S_{A^{-1}}\left(H_{g}\right)$. According to the definition of the sesquilinear form $\langle$,$\rangle we have:$

$$
\langle\tau(x), \tau(y)\rangle_{A^{-1}}=\langle\tau(x \sqcup y)\rangle_{A^{-1}}=\langle x \sqcup y\rangle_{A^{-1}}
$$

It follows that $x \in \operatorname{ker}\langle,\rangle_{A}$ if and only if $\tau(x) \in \operatorname{ker}\langle,\rangle_{A^{-1}}$. We obtain $W_{g, p,(i)}$ as the quotient by the kernel of $\langle,\rangle_{A}$ after specifying an embedding $\mathbb{Z}\left[A, A^{-1}\right] \rightarrow \mathbb{C}$ sending $A$ to a $2 p$-th root of unity. It follows that $\tau$ induces the complex conjugation at the level of $W_{g, p,(i)}$. In other terms $\tau$ provides an isomorphism between the space of conformal blocks $W_{g, p,(i)}$ associated to the surface $\Sigma_{g,(i)}$ and its dual $W_{g, p,(i)}^{*}$ which is associated to the surface $-\Sigma_{g,(i)}$ with the opposite orientation.

Remark 4.5. More generally, kernels of unitary TQFT representations are characteristic subgroups of $\pi_{g}$.

4.5. Generalized congruence quotients. A principal congruence subgroup of $\Gamma_{g}^{1}$ is the kernel of the homomorphism $\operatorname{Aut}^{+}\left(\pi_{g}\right) \rightarrow \operatorname{Aut}(F)$ induced by some surjective homomorphism $\pi_{g} \rightarrow F$ onto a finite characteristic quotient $F$ of $\pi_{g}$. We actually only need an $\operatorname{Aut}^{+}\left(\pi_{g}\right)$-invariant quotient $F$. The image of $\operatorname{Aut}^{+}\left(\pi_{g}\right)$ within $\operatorname{Aut}(F)$ is called a principal congruence quotient. This construction naturally extends to all characteristic quotients $F$ of $\pi_{g}$, not necessarily finite ones; we will call them generalized principal congruence subgroups and quotients respectively.

Proposition 4.3. If $\left.\rho_{p,(i)}\right|_{\pi_{g}}: \pi_{g} \rightarrow P \mathbb{G}_{g, p,(i)}$ is Zariski dense then the mapping class group representation $\rho_{p,(i)}$ is equivalent to a generalized principal congruence representation. In particular, this holds when $i=p-3$.

Proof. The action by conjugacy of $\rho_{p,(i)}\left(\Gamma_{g}^{1}\right)$ on $\rho_{p,(i)}\left(\pi_{g}\right)$ provides a homomorphism:

$$
\Phi: \rho_{p,(i)}\left(\Gamma_{g}^{1}\right) \rightarrow \operatorname{Aut}\left(\rho_{p,(i)}\left(\pi_{g}\right)\right)
$$

In the proof of Lemma 4.2 we noted that $\rho_{p,(i)}\left(\pi_{g}\right)$ are characteristic quotients of $\pi_{g}$. It remains to prove that the homomorphism $\Phi$ is injective. Let $\varphi \in \Gamma_{g}^{1}$ such that $\rho_{p,(i)}(\varphi) \in \operatorname{ker} \Phi$. This is equivalent to:

$$
\rho_{p,(i)}(\varphi) \rho_{p,(i)}(x) \rho_{p,(i)}(\varphi)^{-1}=\rho_{p,(i)}(x), \text { for all } x \in \pi_{g}
$$

Since $\rho_{p,(i)}\left(\pi_{g}\right)$ is Zariski dense in $P \mathbb{G}_{g, p,(i)}$ this implies that $\rho_{p,(i)}(\varphi)$ is identity. This proves the claim.

Remark 4.6. The same proof works under the weaker assumption that $\left.A d \circ \rho_{p,(i)}\right|_{\pi_{g}}$ is irreducible (see [39], Prop.14). Moreover, if we suppose that $\left.\rho_{p,(i)}\right|_{\pi_{g}}$ is irreducible, then the homomorphism $\Phi$ has a finite abelian kernel (see [39], Prop.15).

Proposition 4.4. The finite quotients $P \mathbb{G}_{g, p,(p-3)}\left(\mathbb{Z} / q^{k} \mathbb{Z}\right)$ of $\Gamma_{g}^{1}$ obtained in Proposition 4.1 are principal congruence quotients. 
Proof. The proof of Theorem 1.4 and Proposition 4.3 above show that the image of $\Gamma_{g}^{1}$ acts by conjugacy on the finite characteristic quotient of $\pi_{g}$ obtained by reduction $\bmod q^{k}$ of $\rho_{p,(p-3)}\left(\pi_{g}\right)$. The reduction $\bmod q^{k}$ of the map $\Phi$ above is still injective since $P \mathbb{G}_{g, p,(p-3)}(\mathbb{Z} / q \mathbb{Z})$ are centerfree.

4.6. Comments. The statement of Theorem 1.4 had several other reformulations which were discussed in $(22,6.4)$. Conjecture 6.12 from [22] claims that for all finite dimensional linear representations of the group Aut $\left(\mathbb{F}_{n}\right)$ of automorphisms of the free group $\mathbb{F}_{n}$ on $n \geq 3$ generators the image of the subgroup $\mathbb{F}_{n}$ of inner automorphisms is virtually solvable. Proposition 3.2 above shows that a similar statement cannot hold when the free group $\mathbb{F}_{n}$ is replaced by a surface group $\pi_{g}, g \geq 2$. This already follows from ([18], Cor. 4.2). Although representations $\widetilde{\rho}_{g, p,(i)}$ arising here are all projective representations, they can be easily converted into linear representations with the same properties by considering the tensor product $\widetilde{\rho}_{g, p,(i)} \otimes \widetilde{\rho}_{g, p,(i)}^{*}$ with their respective dual representations. This conjecture is both related to the non-linearity of $\operatorname{Aut}\left(\mathbb{F}_{n}\right)$, for $n \geq 3$ following Formanek and Procesi and the Weigold conjecture. The later states that Aut $\left(\mathbb{F}_{n}\right)$ acts transitively on the set of kernels of surjective homomorphisms $\mathbb{F}_{n} \rightarrow G$, for any finite simple group $G$ (see [22]) and it is known to hold for large enough $n$ in terms of $G$.

In ([22], section 10) the authors discussed similar questions for a surface group. Any homomorphism $\pi_{g} \rightarrow G$ defines a class in $H_{2}(G)$ which is the image of the fundamental class of $H_{2}\left(\pi_{g}\right)$. This class is left invariant by the left composition with automorphisms from $\operatorname{Aut}\left(\pi_{g}\right)$ and its image in $H_{2}(G) / \operatorname{Out}(G)$ is also invariant by the right composition with automorphisms from Aut $(G)$. The extended Weigold conjecture asks whether $\operatorname{Aut}\left(\pi_{g}\right)$ (or just $\operatorname{Aut}^{+}\left(\pi_{g}\right)$ ) acts transitively on the set of kernels of surjective homomorphisms $\pi_{g} \rightarrow G$ corresponding to a given class in $H_{2}(G) / \operatorname{Out}(G)$, provided $G$ is a finite simple group and $g \geq 3$. This was proved to hold for large enough $g$, depending on $G$ in $[\underline{6}$.

The existence of characteristic finite simple quotients of $\pi_{g}, g \geq 2$ from Theorem 1.4 and the discussion in $([22,6.4)$ also show that this analog of the Weigold conjecture for surface groups does not hold. We choose $p$ such that $N_{g, p}$ are odd and then primes $q$ such that $q-1$ is coprime to $N_{g, p}$. Then $H_{2}\left(P S L\left(N_{g, p}, \mathbf{F}_{q}\right)\right)=1$, so that there is only one class of homomorphisms. One knows from [6] that there exists a large orbit of $\operatorname{Aut}\left(\pi_{g}\right)$ where this group acts as an alternating group. On the other hand for infinitely many finite quotients of $\pi_{g}$ of the form $P S L\left(N_{g, p}, \mathbf{F}_{q}\right)$ the action of $\operatorname{Aut}\left(\pi_{g}\right)$ is trivial. Thus for large enough $q$ there exist several $\operatorname{Aut}\left(\pi_{g}\right)$-orbits (in the same class).

Another problem stated in $([22,6.4)$ for the free group is whether, for a Chevalley group scheme $F$ and surjective homomorphisms $\Psi: \pi_{g} \rightarrow F\left(\mathbf{F}_{q}\right)$ the number of conjugacy classes in the image $\Psi\left(\mathcal{S}\left(S_{g}\right)\right)$ of primitive elements (i.e. simple closed curves) is unbounded as $q \rightarrow \infty$. For those $\Psi$ encountered in the proof of Theorem 1.4 the image of $\Gamma_{g}^{1}$ is still $F\left(\mathbf{F}_{q}\right)$ and hence there are at most $\left[\frac{g}{2}\right]+1$ conjugacy classes for all $q$.

\section{REFERENCES}

[1] J. E. Andersen, Asymptotic faithfulness of the quantum $\mathrm{SU}(n)$ representations of the mapping class groups, Ann. of Math. 163(2006), 347-368.

[2] C. Blanchet, N. Habegger, G. Masbaum and P. Vogel, Topological quantum field theories derived from the Kauffman bracket, Topology 34 (1995), 883-927.

[3] A. Borel and Harish-Chandra, Arithmetic subgroups of algebraic groups, Ann. of Math. (2) 75 (1962), 485-535.

[4] J. Dieudonné, On the automorphisms of the classical groups, Memoirs Amer. Math. Soc. no.2, 1950.

[5] N. Dunfield and H. Wong, Quantum invariants of random 3-manifolds, Algebr. Geom. Topol. 11 (2011), no. 4, 2191-2205.

[6] N. Dunfield and W. Thurston, Finite covers of random 3-manifolds, Invent. Math. 166(2006), 457-521.

[7] G. Faltings, Endlichkeitssätze für abelsche Varietäten über Zahlkörpern, Invent. Math. 73 (1983), 349-366, Erratum $75(1984), 381$.

[8] M. H. Freedman, M. Larsen and Z. Wang, The two-eigenvalue problem and density of Jones representation of braid groups, Comm. Math. Phys. 228 (2002), 177-199.

[9] M. H. Freedman, K. Walker and Z. Wang, Quantum SU(2) faithfully detects mapping class groups modulo center, Geom. Topol. 6 (2002), 523-539.

[10] L. Funar, On the TQFT representations of the mapping class groups, Pacific J. Math. 188 (2)(1999), 251-274.

[11] L. Funar, Zariski density and finite quotients of mapping class groups, International Mathematics Research Notices 2013, no.9, 2078-2096. 
[12] L. Funar and W. Pitsch, Images of quantum representations of mapping class groups and Dupont-GuichardetWigner quasi-homomorphisms, arxiv 1209.0302, J. Inst. Math. Jussieu, to appear.

[13] L. Funar and T. Kohno, On Burau representations at roots of unity, Geometriae Dedicata 169(2014), $145-163$.

[14] S. Gervais, Presentation and central extensions of mapping class groups, Trans. Amer. Math. Soc. 348 (1996), 3097-3132.

[15] P. Gilmer and G. Masbaum, Integral lattices in TQFT, Ann. Sci. Ecole Norm. Sup. (4) 40 (2007), 815-844.

[16] P. Gilmer and G. Masbaum, Irreducible factors of modular representations of mapping class groups arising in Integral TQFT, Quantum Topology, 5 (2014), 225-258.

[17] P. Hall, The Eulerian functions of a group, Quarterly J. Math. 7(1936), 134-151.

[18] T. Koberda, R. Santharoubane, Quotients of surface groups and homology of finite covers via quantum representations, Invent. Math. 206(2016), 262-292.

[19] G. Kuperberg, Denseness and Zariski denseness of Jones braid representations, Geom. Topol. 15 (2011), 11-40.

[20] S. Lang, Elliptic curves: Diophantine analysis, Grundlehren der mathematischen Wissenschaften 231, Springer Verlag, Berlin Heidelberg, 1978.

[21] M. Larsen and Z. Wang, Density of the SO(3) TQFT representation of mapping class groups, Comm. Math. Phys. 260 (2005), 641-658.

[22] A. Lubotzky, Dynamics of $\operatorname{Aut}\left(F_{n}\right)$ actions on group presentations and representations, "Geometry, Rigidity, and Group Actions" pp. 609-643, a collection of papers dedicated to Bob Zimmer, edited by B. Farb and D. Fisher, Chicago University Press, 2011.

[23] J. Marché and M. Narimannejad, Some Asymptotics of TQFT via skein theory, Duke Math. J. 141 (2008), $573-587$.

[24] J. Marché and L. Charles, Multicurves and regular functions on the representation variety of a surface in SU(2), Commentarii Math. Helv. 87 (2012), 409-431.

[25] J. Marché, personal communication.

[26] G. A. Margulis, Discrete subgroups of semisimple Lie groups, Ergebnisse der Mathematik und ihrer Grenzgebiete (3), 17, Springer-Verlag, Berlin-New York, 1991, x+388 pp.

[27] G. Masbaum, An element of infinite order in TQFT-representations of mapping class groups, Low-dimensional topology (Funchal, 1998), 137-139, Contemp. Math., 233, Amer. Math. Soc., Providence, RI, 1999.

[28] G. Masbaum and A. Reid, All finite groups are involved in the mapping class groups, Geom.Topol. 16 (2012) $1393-1411$.

[29] G. Masbaum and J. Roberts, On central extensions of mapping class groups, Math. Ann. 302 (1995), 131-150.

[30] M. Nori, On subgroups of $G L_{n}\left(\mathbb{F}_{p}\right)$, Invent. Math. 88 (1987), 257-275.

[31] V. Platonov and A. S. Rapinchuk, Algebraic Groups and Number Fields, Pure and Appl. Math Monographs 139, Academic Press 1994.

[32] G. Prasad and A.S. Rapinchuk, Developements on the congruence subgroup problem after the work of Bass, Milnor and Serre, in "Collected papers of John Milnor", vol. V, Amer. Math. Soc. (2010), 307-325.

[33] L. Ribes and P. Zalesskii, Profinite groups, second edition. 40, Series of Modern Surveys in Math., 2010.

[34] C.E. Rickart, Isomorphic groups of linear transformations. II., Amer. J. Math. 73 (1951), 697-716.

[35] J.Roberts, Irreduciblity of some quantum representations of mapping class groups, J.Knot Theory Ramif. 10 (2001), 763-767.

[36] P. Scott, Subgroups of Surface Groups Are Almost Geometric, J. London Math. Soc. 2 (17) (1978), 555-565.

[37] P. Scott, Correction to Subgroups of Surface Groups Are Almost Geometric, J. London Math. Soc. 2 (32) (1985), 217-220.

[38] J.-P. Serre, Abelian l-adic representations and elliptic curves, AddisonWesley Publ. Co., 1989.

[39] A. S. Sikora, Character varieties of abelian groups, Trans. Amer. Math. Soc. 364 (2012), 5173-5208.

[40] J. Tits, Classification of algebraic semisimple groups, in Algebraic Groups and Discontinuous Subgroups, Proc. Sympos. Pure Math., 9, 33-62, Amer. Math. Soc., 1966.

[41] G. Tomanov, On the congruence-subgroup problem for some anisotropic algebraic groups over number fields, Journal reine angew. Math. 402 (1989), 138-152.

[42] A. Vasiu, Surjectivity criteria for p-adic representations I, Manuscripta Math. 112 (2003), 325-355.

[43] John H. Walter, Isomorphisms between projective unitary groups, Amer. J. Math. 77 (1955), 805-844.

[44] B. Weisfeiler, Strong approximation for Zarsiki-dense subgroups of semi-simple algebraic groups, Ann. of Math. 120(1984), 271-315.

[45] E. Zelmanov, On some open problems related to the restricted Burnside problem, Contemporary Mathematics 224, 1999, 237-243.

Institut Fourier, Laboratoire de Mathematiques UMR 5582, Université Grenoble Alpes, CS 40700, 38058 Grenoble, France

E-mail address: louis.funar@univ-grenoble-alpes.fr

Centre de Mathématiques de Jussieu, Université Paris Pierre et Marie Curie 4, Place Jussieu, F-75252 PARIS CEDEX 05, FrANCE

E-mail address: pierre.lochak@imj-prg.fr 